MPP-2015-248

FPAUO-15/15

\title{
Entanglement Entropy in a Holographic Kondo Model
}

\author{
Johanna Erdmenger ${ }^{a}, 1$, Mario Flory ${ }^{a} 2$, Carlos $\left.\operatorname{Hoyos}^{b}\right\}^{3}$ \\ Max-Niklas Newrzella ${ }^{a} 4$ and Jackson M. S. Wu ${ }^{c, d: 5}$ \\ ${ }^{a}$ Max-Planck-Institut für Physik (Werner-Heisenberg-Institut), \\ Föhringer Ring 6, D-80805 Munich, Germany. \\ ${ }^{b}$ Department of Physics, Universidad de Oviedo, \\ Avda. Calvo Sotelo 18, 3300\%, Oviedo, Spain. \\ ${ }^{c}$ Department of Physics and Astronomy, University of Alabama, \\ Tuscaloosa, AL 35487, USA. \\ ${ }^{d}$ National Center for Theoretical Sciences, Physics Division, \\ No. 101, Section 2, Kuang Fu Road, Hsinchu, Taiwan 300, R. O. C.
}

\begin{abstract}
We calculate entanglement and impurity entropies in a recent holographic model of a magnetic impurity interacting with a strongly coupled system. There is an RG flow to an IR fixed point where the impurity is screened, leading to a decrease in impurity degrees of freedom. This information loss corresponds to a volume decrease in our dual gravity model, which consists of a codimension one hypersurface embedded in a BTZ black hole background in three dimensions. There are matter fields defined on this hypersurface which are dual to Kondo field theory operators. In the large $N$ limit, the formation of the Kondo cloud corresponds to the condensation of a scalar field. The entropy is calculated according to the Ryu-Takayanagi prescription. This requires to determine the backreaction of the hypersurface on the BTZ geometry, which is achieved by solving the Israel junction conditions. We find that the larger the scalar condensate gets, the more the volume of constant time slices in the bulk is reduced, shortening the bulk geodesics and reducing the impurity entropy. This provides a new non-trivial example of an RG flow satisfying the $g$-theorem. Moreover, we find explicit expressions for the impurity entropy which are in agreement with previous field theory results for free electrons. This demonstrates the universality of perturbing about an IR fixed point.
\end{abstract}

Dedicated to the memory of our colleague Peter Breitenlohner

\footnotetext{
1 jke@mpp.mpg.de

2 mflory@mpp.mpg.de

3 hoyoscarlos@uniovi.es

4 maxnew@mpp.mpg.de

5 jknw350@yahoo.com
} 


\section{Contents}

1 Introduction and Summary 1

2 Review: A Holographic Kondo Model 6

2.1 Action and geometric setup . . . . . . . . . . . . . . 6

2.2 Equations of motion and boundary conditions . . . . . . . . . . . . . 10

2.3 The normal phase . . . . . . . . . . . . . . . . . . 13

3 Entanglement Entropy in the Holographic Model 14

3.1 Numerical results . . . . . . . . . . . . . . . . . . . . . . . . . . 14

3.2 Holographic $g$-theorem . . . . . . . . . . . . . . . . . . . 17

3.3 Large $\ell$ approximation . . . . . . . . . . . . . . . . . . . . 19

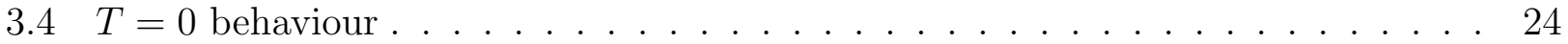

4 Conclusions and Outlook 25

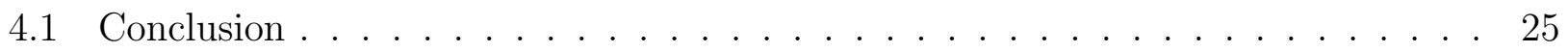

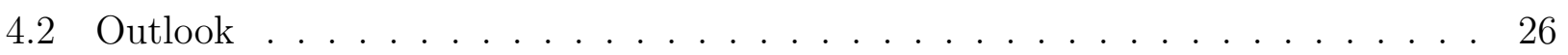

A Junction Conditions for Abelian Chern-Simons Fields 27

B Details of Numerics

\section{Introduction and Summary}

The Kondo model, proposed by Jun Kondo in 1964 [1], describes the interaction of a single magnetic impurity with the fermionic quasi-particles of a Landau Fermi Liquid (LFL). The Kondo Hamiltonian includes two terms: a kinetic term for the LFL quasi-particles, and a Kondo coupling between the LFL spin current and the impurity's spin.

The Kondo model was seminal in many respects. For example, the Kondo coupling constant approaches zero in the ultraviolet (UV), appears to diverge at a dynamically-generated scale and in the infrared (IR), conventionally expressed as a temperature, the Kondo temperature, $T_{\mathrm{K}}$. The Kondo model thus provided an example of asymptotic freedom and dimensional transmutation pre-dating Quantum Chromodynamics (QCD) by almost ten years. Moreover, the Kondo model was seminal to Wilson's development of (numerical) Renormalization Group (RG) techniques [2 4], integrability [5 12], large- $N$ limits [13 18], Conformal Field Theory (CFT) techniques [16, 19 24], and more [25,26].

The "Kondo problem" is to find the eigenstates of the Kondo Hamiltonian for all values of temperature $T$, and from them to compute all observables. The Kondo problem is considered solved, via a combination of the techniques mentioned above. The solution reveals the "Kondo effect", that is, the screening of the impurity spin by the LFL when $T \lesssim T_{\mathrm{K}}$. In particular, the LFL quasi-particles form a screening cloud around the impurity, the "Kondo cloud", which 
at $T=0$ has a characteristic size, $\xi_{\mathrm{K}}=v / T_{\mathrm{K}}$ where $v$ is the Fermi velocity. For a dilute concentration of magnetic impurities, the Kondo effect leads to a characteristic $-\ln \left(T / T_{\mathrm{K}}\right)$ contribution to the resistivity [1]. At $T \approx T_{\mathrm{K}}$, perturbation theory breaks down.

Although the Kondo problem is considered solved, many questions about the original Kondo model actually remain very challenging, such as the the dependence of the Entanglement Entropy (EE) on the size of a spatial sub-system [27-30], the effect of a quantum quench of the Kondo coupling [31], or how to define and measure $\xi_{K}$ precisely [32]. More generally, a major challenge is to solve the Kondo problem when the LFL is replaced by strongly-interacting degrees of freedom, including in particular, in one spatial dimension, a Luttinger liquid.

The Anti-de Sitter/CFT (AdS/CFT) correspondence [33 35], also known as holography, may be uniquely well-suited to address many of these open questions. AdS/CFT equates a weaklycoupled theory of gravity in $(d+1)$-dimensional AdS spacetime, $A d S_{d+1}$, with a strongly-coupled $d$-dimensional CFT "living" on the boundary of $A d S_{d+1}$. Typically the strongly-coupled CFT is a non-Abelian gauge theory in the 't Hooft large- $N$ limit [36, 37.

Various holographic impurity models have been proposed: see for example refs. [38 48]. In all of these models, the global $S U(2)$ spin symmetry is replaced by an $S U(N)$, which is then gauged, that is, $S U(N)$ gauge fields were introduced, and possibly additional fields, such as supersymmetric partners of the gauge fields. The impurity is then described as an $S U(N)$ Wilson line [49 54]. Gauging $S U(N)$ introduces an additional coupling, the 't Hooft coupling. All of the models in refs. [38 48] employed the 't Hooft large- $N$ limit and large 't Hooft coupling. In other words, all of the holographic models in refs. [38 48 are strongly-interacting mean-field models.

However, most of the holographic models in [38 48] describe only fixed points. Indeed, only the holographic model of [48] describes an RG flow with a Kondo coupling that exhibites asymptotic freedom and the appearance of $T_{\mathrm{K}}$. The new aspects of [48] include a holographic description of the flow from a UV to an IR fixed point triggered by a 'double-trace' marginally relevant operator and a gravity dual of the screening mechanism. The essential difference between this holographic model and the original Kondo model is that instead of the free electron gas, there is an inherently strongly coupled system which interacts with the magnetic impurity. Moreover, just as in the models $[38-48]$ the gauge/gravity duality approach requires a large $N$ limit for the $S U(N)$ spin group in $[48$. Due to this limit, the characteristic logarithmic rise of the resistivity at low temperatures is absent. Instead, a leading irrelevant operator analysis shows that the resistivity scales with temperature as $T^{\Delta}, \Delta$ being a real number greater than one. This is reminiscent of a Luttinger liquid behaviour, and an interesting task for the future will be to determine the Luttinger parameter for the holographic model proposed in [48].

The large $N$ Kondo model was studied in field theory (with free electrons and a global $S U(N)$ spin group) already some time ago [55,56]. As was noted there, the model simplifies considerably by introducing slave fermions $\chi$ and writing the impurity spin operator $\boldsymbol{S}$ as $S^{a}=\bar{\chi} T^{a} \chi$. $T^{a}$ is a generator of $S U(N)$ in the fundamental representation. $\boldsymbol{S}$ is in a totally antisymmetric representation, corresponding to a Young tableau with $Q$ boxes. The chiral fermions are constrained by the fact that their charge density has to be equal to $Q$. The crucial property of 
the large $N$ Kondo model is that the screening corresponds to a condensation of the operator $\mathcal{O}=\psi^{\dagger} \chi$, where $\psi$ is an electron field and $\chi$ the slave fermion. The condensate breaks the $U(1) \times U(1)$ symmetry of the electrons and slave fermions to the diagonal $U(1)$. In the large $N$ limit, long-range fluctuations are suppressed, such that condensate formation is possible also in two dimensions. There is a phase transition at a critical temperature $T=T_{c}$ in the large $N$ Kondo model, whereas for finite $N$ the transition is a crossover.

This condensation has a very natural analogue in gauge/gravity duality, in the form of a holographic superconductor in $\mathrm{AdS}_{2}$. Motivated by a top-down brane construction which realises the scalar dual to the operator $\mathcal{O}=\psi^{\dagger} \chi$ as strings streching from D5 to D7-branes, in [48] a bottom-up holographic Kondo model was suggested, in which the electron current is dual to an $\mathrm{AdS}_{3}$ Chern-Simons field $A_{\mu}$, in addition to the scalar field dual to $\mathcal{O}$ which lives on an $\mathrm{AdS}_{2}$ subspace. Moreover, the charge density dual is given by the temporal component $a_{t}$ of a gauge field on $\mathrm{AdS}_{2}$. Finite temperature is introduced by considering a BTZ black hole background instead of $\mathrm{AdS}_{3}$. In this model, the defect $\mathrm{RG}$ flow is generated by the gravity dual of the product operator $\mathcal{O O}^{\dagger}$, which is obtained in analogy to the holographic double-trace deformation proposed in [57]. This RG flow displays the required scale generation. Moreover, at low energies $\mathcal{O}$ condenses. This gives rise to a screening of the impurity, as may be checked by calculating the flux of $a_{t}$ in the $\mathrm{AdS}_{2}$ subspace. At the boundary, this flux coincides with $Q$. At the horizon, this flux decreases when lowering the temperature, such that there are less impurity degrees of freedom visible.

In this paper, in view of providing further comparison with field theory results, we calculate the entanglement entropy for the model described above. The application of the Ryu-Takayanagi (RT) approach [58,59] for the holographic entanglement entropy requires to include the effect of the backreaction of the $\mathrm{AdS}_{2}$ hypersurface on the $\mathrm{AdS}_{3}$ geometry. For a simple holographic model which describes the backreaction of the defect on the surrounding geometry, we follow 6063 and consider a hypersurface with the required matter content, i.e. a two-dimensional gauge field and a complex scalar. The geometry is then determined by the Israel junction conditions. As discussed in [63], these conditions are influenced by the energy conditions for the brane matter fields. In this paper, we will often refer to the hypersurface as "brane", as it is the remnant of the intersection of the D5 and D7-branes studied in the top-down model of [48]. The geometry remains BTZ everywhere except at the locus of this brane. The effect of the Israel junction conditions is to cut out parts of the bulk volume, in dependence on the brane tension generated by the brane matter fields.

We calculate the entanglement entropy in this geometry as follows: The field theory entangling region is an interval of length $2 \ell$ symmetrically located on both sides of the impurity. Then, according to the Ryu-Takayanagi prescription, the entanglement entropy is given by the length of the bulk geodesic with endpoints coinciding with the endpoints of the boundary interval. Via the Israel junction conditions implementing the backreaction, the matter fields on the brane affect the length of this geodesic, and thus the entanglement entropy. 
Within field theory, the impurity entropy is defined by the difference [27-30]

$$
\left.S_{\text {imp }}(\ell) \equiv S(\ell)\right|_{\text {Impurity present }}-\left.S(\ell)\right|_{\text {Impurity absent }} .
$$

This quantity was argued to provide a good description of the Kondo cloud profile [30]. We use the same definition for the impurity entropy in our holographic computation. The subtraction guarantees a finite result in the holographic approach.

A central result of our holographic calculation is that as the condensate $\langle\mathcal{O}\rangle=\left\langle\chi^{\dagger} \psi\right\rangle$ increases, the spacetime volume shrinks. This results in shorter geodesics normal to the brane and hence in smaller impurity entropy. This is in agreement with field theory expectations, since in the large $N$ limit, the formation of the $\langle\mathcal{O}\rangle$ condensate corresponds to screening of the impurity. When the impurity is screened, less impurity degrees of freedom are visible, which is indeed the case in our holographic approach. This physical property is encoded in the $g$-theorem [22, 64]. Indeed, our model adds one more example to the explicit realizations of boundary RG flows manifestly satisfying the $g$-theorem. A field theory example for such a flow may be found in [65]. For further examples within holography, see $[60,61,66]$. We also note that our holographic map between the decrease of entanglement entropy and the decrease of volume in the dual gravity theory may be related to recent discussions of quantum complexity in the holographic context 67-71].

The agreement with field theory results may be made precise at least in the large $\ell$ and small $T$ limit. The impurity entropy was calculated both using density matrix renormalisation group (DMRG) and CFT approaches [27 30] for free electrons coupled to a magnetic impurity. The $T=0$ result is $[27-30]$

$$
S_{i m p}=\frac{\pi c}{12} \frac{\xi_{\mathrm{K}}}{\ell} \text { for } \ell \gg \xi_{\mathrm{K}}
$$

Here $c$ is the central charge. In [29] this result was generalised to small but non-zero temperature,

$$
S_{i m p}=\frac{\pi^{2} c \xi_{\mathrm{K}} T}{6 v} \operatorname{coth}\left(\frac{2 \pi \ell T}{v}\right)=\frac{\pi^{2} c}{6} \frac{T}{T_{\mathrm{K}}} \operatorname{coth}\left(2 \pi \frac{\ell}{\xi_{\mathrm{K}}} \frac{T}{T_{\mathrm{K}}}\right) \text { for } T / T_{\mathrm{K}}, \quad \xi_{\mathrm{K}} / \ell \ll 1 .
$$

We find that a similar result is obtained in the holographic approach, where the field theory is strongly coupled. This is achieved in the following way: An RG flow is given by the defect brane which reaches from the boundary to the horizon. Since the energy-momentum tensor originating from the defect matter fields varies as function of the radial coordinate, the brane bends. It asymptotes to a constant tension brane in the UV near the boundary, i.e. the energymomentum tensor on the brane corresponds to constant tension. Near the horizon, the energymomentum tensor on the brane also asymptotes to constant tension form, however with a different tension than in the UV. This corresponds to an IR fixed point. We consider a geometric linear perturbation about this fixed point by fitting a constant tension brane to the asymptotic behaviour of the brane at the horizon. Extrapolating this constant tension brane to the UV, 
it intersects the AdS boundary at a distance $D$ from the impurity, see figure 1. The impurity entropy (1) then takes the form

$$
S_{i m p}(\ell)=S_{\mathrm{BH}}(\ell+D)-S_{\mathrm{BH}}(\ell)
$$

with $S_{\mathrm{BH}}$ the Ryu-Takayanagi entanglement entropy for the BTZ black hole. Expanding to linear order in $D$, we recover the field theory result (3) provided that we identify

$$
D \propto \xi_{\mathrm{K}},
$$

i.e. $D$ plays the role of the Kondo correlation length. This remarkable agreement may be traced back to the fact that in both field theory and holography, perturbation about a CFT at an IR fixed point is involved, which induces universal behaviour independently of the precise form of the theory at the fixed point.

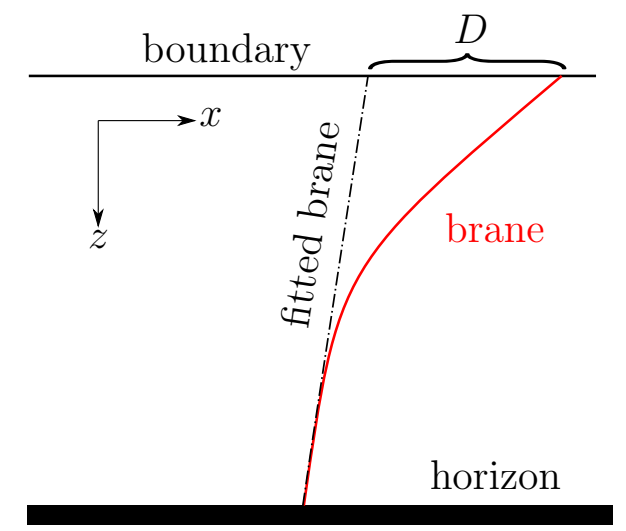

Figure 1: Near horizon approximation of the brane via a constant tension solution. See section 3.3 for details, and section 2.1 for the geometric construction of the backreaction of the brane.

The structure of this paper is as follows: In section 2 we summarise the most important features of our holographic Kondo model as presented in [48,63]. In particlular, we explain how the defect on the boundary is described by a thin brane in the bulk, and how the Israel junction conditions describe the backreaction of this brane on the geometry. In section 3.1 we present numerical results for the backreaction and entanglement entropy in our Kondo model. We then interpret these results, describing both the reduction of the impurity entropy and spacetime volume (interpreted as holographic complexity [67-71]) as signs of the screening of the impurity by the Kondo cloud. Furthermore, we comment on the holographic $g$-theorem in section 3.2 . and compare our numerical and semi-analytical results to the field theory result (3) in section 3.3. As we will see, the null energy condition (NEC) plays a pivotal role throughout these discussions. Special attention is also given to the behaviour at $T=0$ in section 3.4. We then end in section 4 with a conclusion and outlook. In appendix $A$ we comment on how to include the bulk Chern-Simons field in our calculations, and in appendix B we give some details on the numerical methods employed to solve the equations of motion. 


\section{Review: A Holographic Kondo Model}

\subsection{Action and geometric setup}

In this section we will briefly review the holographic model of the Kondo effect proposed in [48, and the approach to including backreaction in this model outlined in [63]. For more details about this model, see those two papers. Results on the entanglement entropy obtained from this model will be discussed below in section 3 ,

In [48], a bottom-up holographic model was motivated by a top-down string construction involving a background of $N$ D3-branes as well as $k$ probe D7-branes and one probe D5-brane. The probe D7-branes are embedded in such a way as to lead to chiral fermions in $1+1$ dimensions [72 75], which may be interpreted as electrons in $3+1$ dimensions (for the interaction with the defect, the s-wave approximation is used, as is standard in the CFT approach to the Kondo effect). Note that the string construction makes them inherently chiral. From the field theory point of view, these chiral fermions correspond to a CFT with Kac-Moody algebra $S U(N)_{k} \times S U(k)_{N}$. Note that the $S U(N)_{k}$ symmetry is gauged in this approach. The gauge anomaly is suppressed in the probe limit $k / N \rightarrow 0$. The probe D5-brane introduces the $(0+1)$-dimensional slave fermions $\chi$ as in $51,53,72$. In the dual gravity model, the D5-brane leads to the instability inducing the condensation process.

Motivated by the above top-down construction, we now turn to a simpler bottom-up model which allows to study the defect backreaction by considering a thin brane. On the gravity side, this model contains both $(2+1)$-dimensional bulk fields and $(1+1)$-dimensional defect fields, where the defect spans the time and bulk radial directions. The starting point is a $(2+1)$-dimensional Einstein-Hilbert contribution to the action,

$$
\mathcal{S}_{g}=\frac{1}{2 \kappa_{N}^{2}} \int d^{3} x \sqrt{-g}(R-2 \Lambda)
$$

with $R$ the Ricci scalar and the negative cosmological constant $\Lambda=-1 / L^{2}, L$ being the AdS

radius. In the absence of the defect allows for the BTZ black hole solution 76, 77, with metric

$$
\mathrm{d} s^{2}=\frac{L^{2}}{z^{2}}\left(-f(z) \mathrm{d} t^{2}+\frac{\mathrm{d} z^{2}}{f(z)}+\mathrm{d} x^{2}\right),
$$

where $L$ is the AdS radius and $f(z)=1-z^{2} / z_{H}^{2}$ with $z_{H}$ the position of the event horizon. This provides the finite temperature $T=1 / 2 \pi z_{H}$ relevant for the Kondo effect. Moreover, there is a Chern-Simons (CS) field $A_{\mu}$ with action

$$
\mathcal{S}_{A}=-\frac{\mathcal{N}}{4 \pi} \int \operatorname{Tr}\left(A \wedge \mathrm{d} A+\frac{2}{3} A \wedge A \wedge A\right)
$$

defined throughout the (2+1)-dimensional bulk spacetime. Here, the normalization factor $\mathcal{N}$ is proportional to $N$. As in the original model of [48], $N$ corresponds to the rank of the gauged 
spin group $S U(N)$, which is not directly visible in the bottom-up model considered. Only the $S U(k)_{N}$ channel symmetry and an additional $U(1)$ charge symmetry are explicitly realised by the Chern-Simons field. $N$ and $k$ correspond to its level and rank.

We consider a single channel or flavour, $k=1$, such that the Chern-Simons theory is $U(1)$. Moreover, there is a Yang-Mills (YM) field $a_{m}$, as well as a complex scalar field $\Phi$ localised in the worldvolume of a codimension one hypersurface that extends from the boundary to the black hole event horizon. The scalar field is charged under both the CS and YM fields and carries opposite charges $\pm q$. The action contribution for these fields reads

$$
\begin{aligned}
& \mathcal{S}_{a, \Phi}=-\mathcal{N} \int \mathrm{d}^{2} x \sqrt{-\gamma}\left(\frac{1}{4} f_{m n} f^{m n}+\gamma^{m n}\left(\mathcal{D}_{m} \Phi\right)^{\dagger}\left(\mathcal{D}_{n} \Phi\right)+V\left(\Phi \Phi^{\dagger}\right)\right), \\
& \mathcal{D}_{m} \Phi \equiv \partial_{m} \Phi+i q A_{m} \Phi-i q a_{m} \Phi
\end{aligned}
$$

The precise form of the potential will be fixed later. For the single-impurity Kondo model, the Yang-Mills field $a$ has gauge group $U(1)$. 6 While Greek indices $\mu$ of bulk quantities run over $2+1$ dimensions, Latin indices $m$ of hypersurface quantities run over $1+1$ dimensions, i.e. over time and the bulk radial direction. The induced metric on the hypersurface or 'brane' is denoted by $\gamma_{m n}$. The precise form of the potential in $(9)$ will be fixed later. Details on the projection of the CS field to the brane are given in appendix A. As shown in [63], the energy-momentum tensor on the brane worldvolume takes the form

$$
S_{i j}=\frac{\mathcal{N}}{2} \gamma_{i j} f^{m n} f_{m n}+2 \mathcal{N}\left[\left(\mathcal{D}_{(i} \Phi\right)^{\dagger} \mathcal{D}_{j)} \Phi-\frac{1}{2} \gamma_{i j}\left(|\mathcal{D} \Phi|^{2}+V\left(\Phi^{\dagger} \Phi\right)\right)\right]
$$

where we used the antisymmetry of $f_{m n}$ to show $\gamma^{m n} f_{m i} f_{n j}=\frac{1}{2} \gamma_{i j} f^{m n} f_{m n}$ in our setup.

In [48], where backreaction on the geometry was neglected, the embedding of the brane into the background spacetime is given by the function $x(z) \equiv 0$, which we call the trivial embedding. In order to calculate the entanglement entropy in this holographic model following the RyuTakayanagi (RT) approach [58, 59], we have to include the backreaction of the brane matter fields on the geometry. This is necessary in order to study the effect of the brane matter fields on the bulk minimal surface over the entangling region. In the low-dimensional case considered here, the minimal surface entering the RT formula is just a geodesic. Since the Chern-Simons bulk action is topological, the only contribution to the energy-momentum tensor comes from the brane matter fields $a_{m}$ and $\Phi$.

The central point of our construction is that the effect of the brane onto the geometry is taken into account via the Israel junction conditions [79]. The geometric setup underlying these junction conditions is depicted in figure 3 . On the left we see the approach followed in this paper, where standard coordinates are used as in (7). The embedding of the brane is then described by the two functions $x_{ \pm}(z) 7, x_{+}$describes the embedding of the brane with respect to the spacetime on the right side of the brane, while $x_{-}$describes the embedding with respect

6 A holographic model of the two-impurity Kondo effect which uses gauge group $U(2)$ was recently proposed in 78. ${ }^{7}$ In this entire work we are only interested in static setups, hence we neglect a possible time dependence $x_{ \pm}(z, t)$. 


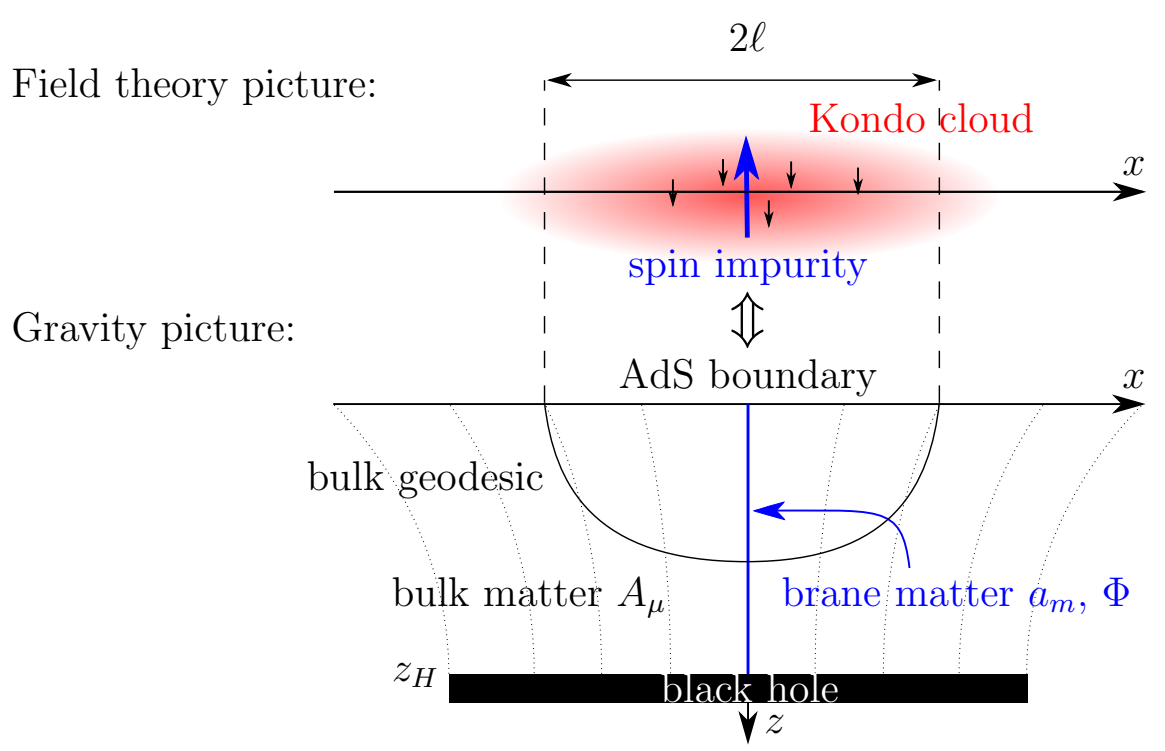

Figure 2: Bulk setup of the holographic Kondo model of [48]. The localised spin impurity on the field theory side is described by a codimension one hypersurface (called 'brane') in the bulk. In this figure, the brane is shown to be trivially embedded, as appropriate for the case without backreaction. We show the boundary interval of length $2 \ell$ for which we calculate the entanglement entropy. The corresponding bulk geodesic used in the Ryu-Takayanagi prescription streches into the bulk and crosses the impurity hypersurface.

to the left side of the spacetime. Corresponding points on these two curves have then to be identified. Hence the part of the spacetime inbetween these two curves is effectively cut out, leading to a reduction of the (regularised) volume of the spacetime. On the right hand side of figure 3, we show an alternative (but equivalent) picture based on Gaussian normal coordinates. In these coordinates, the brane and a neighbourhood on both sides around it can be described by the same coordinate patch (in contrast to the setup depicted on the left side of figure 3). In any case, the spacetime outside the brane is a vacuum solution of Einsteins equations, specifically the BTZ metric (7) in our model. To summarise, we have two BTZ spacetimes glued together at the hypersurface (the brane). Depending on the tension of the matter fields on the brane, a certain part of the ambient BTZ spacetimes is removed, leading to a reduction of the regularised volume.

In the following we assume a symmetric embedding $\left(x_{+}=-x_{-}\right)$, such that the Israel junction conditions take the form

$$
K_{i j}^{+}-\gamma_{i j} K^{+}=-\frac{\kappa_{N}^{2}}{2} S_{i j}
$$

Here $K_{i j}^{+}$is the extrinsic curvature tensor calculated from $x_{+}(z)$, with the convention that the normal vector points from the - side to the + side. The embedding $(t, z) \hookrightarrow\left(t, z, x_{+}(t, z)\right)$ 


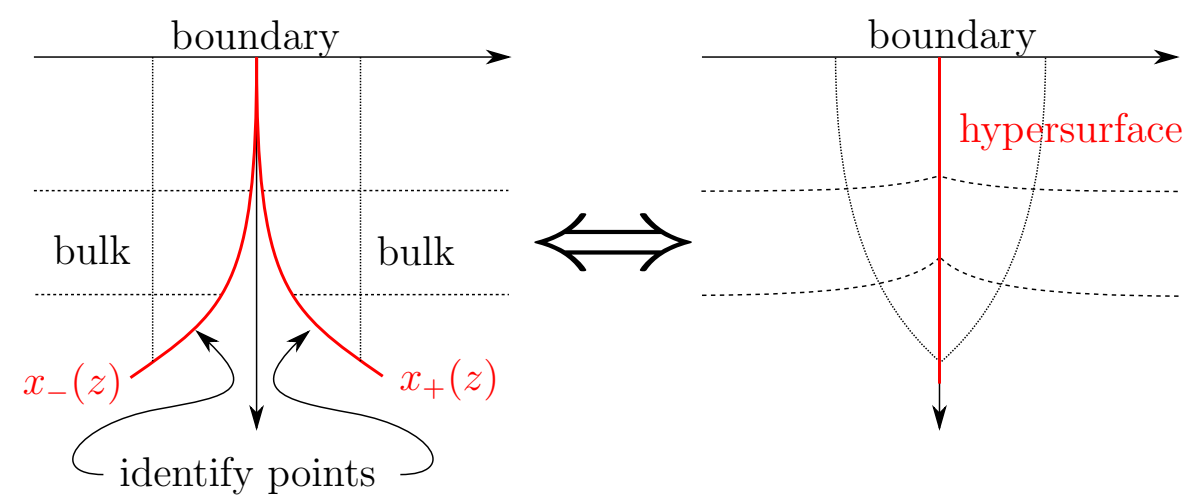

Figure 3: Construction underlying the Israel junction conditions. Left: Construction using standard coordinates as in (7). This is the approach folowed in this paper. Right: It is theoretically possible (albeit too complicated in general) to construct Gaussian normal coordinates around the brane.

induces a metric $\gamma$ on the brane with line element

$$
\mathrm{d} s_{(\gamma)}^{2}=\frac{L^{2}}{z^{2}}\left(-f(z) \mathrm{d} t^{2}+\frac{1+f(z) x_{+}^{\prime}(z)^{2}}{f(z)} \mathrm{d} z^{2}\right) .
$$

The gravitational coupling constant $\kappa_{N}^{2}$ in 12 , which is related to the Newton constant $G_{N}$ by $\kappa_{N}^{2}=8 \pi G_{N}$, is of order $1 / N^{2}$. In order to have non-trivial backreaction on the geometry, we need to rescale all the fields to be of order $\sqrt{N}$, such that effectively $\kappa_{N}^{2} \mathcal{N}$ is of order one, with $\mathcal{N}$ as in (8). This rescaling has to be accompanied by a corresponding rescaling of the charge $q$ in 10 .

A detailed discussion of this construction, as well as further references, may be found in [63]. Moreover, related equations have appeared in the literature already before in holographic studies of boundary CFTs, although through a different geometrical construction. The initial proposal may be found in 6062 . Applications are discussed in 80 87]. It should also be noted that the Israel junction conditions were used also in the study of domain walls and brane worlds, see 88 93.

In [63], some of us derived general results for models described by equation (12), as well as some simple exact solutions for models where the matter content on the brane is simpler than in (9) In section 8 of [63, we also presented some preliminary results on the backreaction in the holographic Kondo model considered also here. There, we neglected the effect of the Chern-Simons field, which we assumed to contribute to the equations of motion with its own junction conditions similar to 12 . Here we verify this assumption by explicitly deriving these

8 A sign difference between $\sqrt{12}$ and the convention used in these papers comes from the differing choice of orientation of the normal vector of the brane, while a factor of $1 / 2$ in our convention comes from the fact that we assume spacetime to be present to both sides of the defect, see figure 3 . See 63 for more details. 9 Specifically, we obtained exact solutions for the cases where the brane matter content is given by a constant tension, a YM-field in the absence of charges, a perfect fluid with equation of state $p=a \cdot \rho, a \geq 0$ and a free massless scalar field, with background spacetimes being either AdS or the non-rotating BTZ black hole. 
junction conditions in appendix A and by showing that the CS field effectively decouples from the dynamics of the brane. This decoupling was implicit in the probe model of [48] through a convenient gauge choice. Moreover, in the present paper, the numerical method and precision is substantially improved as compared to [63]. For detailed information on the numerics, see appendix $\mathrm{B}$ below. In the following, we briefly recapitulate the most important details of the backreacted holographic Kondo model.

\subsection{Equations of motion and boundary conditions}

Above we discussed the Israel junction conditions which are the equations of motions for the embedding scalar $x_{+}$. In the following, we will discuss the equations of motions for the matter fields $\Phi$ and $a$, as well as their asymptotic behaviour. We fix the scalar potential in (9) to be a mass-term only,

$$
V\left(\Phi \Phi^{\dagger}\right)=\frac{1}{2} M^{2} \Phi \Phi^{\dagger},
$$

see section 3.4 for a further discussion of the potential. We choose the radial gauge $a_{z}=0$ and use the residual gauge freedom to set the compex phase, $\psi$, of the scalar field $\Phi \equiv \phi e^{i \psi}$ to zero at some $z$ in the bulk. The radial equation for the gauge field implies that the phase has to vanish everywhere, which must be consistent with the boundary conditions for the scalar field. Hence we pick real boundary conditions for the scalar field. The equation for the $z$-component of the gauge field is then trivially solved. According to the discussion in appendix $\mathrm{A}$, the Chern-Simons field $A$ decouples from the matter confined to the brane, i.e. the equations of motion for $\phi, a_{t}$ and $x_{+}(z)$ can be solved independently. They read

$$
\begin{aligned}
\gamma^{i j} \mathcal{D}_{i} \mathcal{D}_{j} \phi-M^{2} \phi & =0, \\
\partial_{z} \sqrt{-\gamma} f^{z t}+J^{t} & =0, \\
\mathcal{K}_{i j}-\frac{\kappa_{N}^{2}}{2} S_{i j} & =0,
\end{aligned}
$$

where the conserved current is $J^{\mu}=-2 \sqrt{-\gamma} \gamma^{\mu \nu} a_{\nu} \phi^{2}$ and $\mathcal{K}_{i j} \equiv-\left(K_{i j}^{+}-\gamma_{i j} K^{+}\right)$. For the static case, we showed in [63] that by projecting the tensorial equation (17) to its trace and non-trace parts we obtain two scalar equations

$$
\mathcal{K}=\frac{\kappa_{N}^{2}}{2} S \text { and } \mathcal{K}_{L / R}=\frac{\kappa_{N}^{2}}{2} S_{L / R} .
$$

The index $L / R$ signifies the non-trace part and denotes "left/right". This is because while the tensor that projects onto the trace part is by definition the metric $\left(S=\gamma^{i j} S_{i j}\right)$, the tensors that project onto the traceless parts are constructed from the two independent null vectors of the $1+1$-dimensional worldvolume of the brane, one pointing to the "left" $\left(l^{i}\right)$, one pointing to the "right" $\left(r^{i}\right)$, with $l_{i} r^{i} \equiv-1$ and $S_{L} \equiv l^{i} l^{j} S_{i j}, S_{R} \equiv r^{i} r^{j} S_{i j}$. In the static case where there is no energy flux from one side to the other, we can choose a coordinate system such that $S_{L}=S_{R}=S_{L / R}$ is the only component apart from the trace $S$ needed to define $S_{i j}$. 
The two equations in (18) are not independent, but related due to conservation of energymomentum [63]. In particular, from 111$]$ we find

$$
S=\frac{\mathcal{N}}{2} f^{m n} f_{m n}-2 \mathcal{N} V\left(\Phi^{\dagger} \Phi\right), \quad S_{L / R}=\frac{\mathcal{N}}{2} \widehat{\gamma}^{i j}\left(\mathcal{D}_{(i} \Phi\right)^{\dagger} \mathcal{D}_{j)} \Phi
$$

In our choice of coordinates (c.f. (13)), the positive definite tensor $\widehat{\gamma}^{i j}$ coincides with the Euclidean form of the metric $\gamma^{i j}$, see [63]. In order to ensure regularity at the event horizon we have to require

$$
\phi^{\prime}\left(z_{H}\right)=-\frac{L^{2} M^{2} \phi\left(z_{H}\right)}{2 z_{H}}, \quad a_{t}\left(z_{H}\right)=0, \quad x_{+}^{\prime}\left(z_{H}\right)=\kappa_{N}^{2} \mathcal{N} \frac{2 L^{4} M^{2} \phi\left(z_{H}\right)^{2}-z_{H}^{4} a_{t}^{\prime}\left(z_{H}\right)^{2}}{4 L^{3}} .
$$

The condition on the gauge field in the equation above comes from the fact that the Killing vector field $\partial_{t}$ has vanishing norm at the horizon. The other two conditions come from requiring that the most divergent expansion coefficients of the EOMs at the horizon should vanish. Additionally, we need to impose certain conditions at the boundary. The most trivial one is $x_{ \pm}(0)=0$ for the embedding scalar. We can impose this without loss of generality due to the translation invariance of the BTZ metric in the direction of $x$.

Treating the scalar as a probe with respect to the YM field, we find the leading behaviour

$$
a_{t} \sim Q / z+\mu+\ldots
$$

with $Q=-\mathcal{C} L^{2} \cosh (s / L)$ and $\mu=\mu_{c}=\mathcal{C} L^{2} \cosh ^{2}(s / L) / z_{H}$, where the length $s$ parametrises the embedding near the conformal boundary at $z=0$, and $\mathcal{C}$ is the electric flux of the YM field at the conformal boundary. The length $s$ and its relationship with $\mathcal{C}$ will be explained in more detail below in 2.3, in particular in (30). In the top-down construction in [48], it was shown that the electric flux $\mathcal{C}$ coincides with the number of boxes in the Young tableau of the totally antisymmetric representation of the impurity spin $S^{a}=\bar{\chi} T^{a} \chi \cdot \mu$ is identified with the chemical potential for the $U(1)$ charge. For $\mu / T$ larger than a critical value, the scalar condenses.

For the scalar we find two asymptotic solutions near the boundary. As in [48,63], we fix the mass $M$ in exactly such a way that the Breitenlohner-Freedman bound [94] is saturated in order to have the correct operator dimensions needed for a description of the Kondo effect. This yields

$$
M^{2}=\frac{4 Q^{2} \cosh ^{2}(s / L)-1}{4 L^{2} \cosh ^{2}(s / L)}=\left(\frac{Q}{L}\right)^{2}-\left(4 L^{2} \cosh ^{2}(s / L)\right)^{-1} .
$$

This choice ensures that the scalar operator is marginally relevant, such that there is asymptotic freedom. The asymptotic behaviour of the scalar field hence becomes

$$
\phi(z) \sim \alpha \sqrt{z} \log (\Lambda z)+\beta \sqrt{z}+\ldots
$$

where we introduced an arbitrary energy scale $\Lambda$ to define the logarithm. Changing $\Lambda \rightarrow \Lambda^{\prime}$ induces a renormalisation of the dimensionless coupling $\kappa=\alpha / \beta$ given by

$$
\kappa\left(\Lambda^{\prime}\right)=\frac{\kappa(\Lambda)}{1+\kappa(\Lambda) \log \left(\Lambda / \Lambda^{\prime}\right)} .
$$


Next, we exploit two scaling symmetries of the EOMs to set $L=1$ and $z_{H}=1$. Coordinates and fields become dimensionless quantities which we denote by tilded variables

$$
\tilde{z}=z / z_{H}, \quad \tilde{x}=x / z_{H}, \quad \tilde{t}=t / z_{H}, \quad \tilde{\phi}=\phi, \quad \tilde{a}_{t}=a_{t} z_{H}, \quad \tilde{x}_{+}=x_{+} / z_{H}, \quad \text { etc. }
$$

In this new coordinate system we also have

$$
\tilde{a}_{t} \sim Q \frac{z_{H}}{z}+\mu z_{H}+\ldots
$$

and we define $\mu_{T}:=\mu z_{H}=\mu / 2 \pi T$ which effectively labels the solutions for different temperatures. Now

$$
\begin{aligned}
\tilde{\phi}(\tilde{z})=\phi(z) & \sim \alpha \sqrt{z} \log (\Lambda z)+\beta \sqrt{z}+\ldots \\
& =\alpha \sqrt{z_{H}} \sqrt{\tilde{z}} \log (\tilde{z})+\sqrt{\tilde{z}} \sqrt{z_{H}}\left(\beta+\alpha \log \left(\Lambda z_{H}\right)\right) \\
& =: \alpha_{T} \sqrt{\tilde{z}} \log (\tilde{z})+\beta_{T} \sqrt{\tilde{z}}+\ldots
\end{aligned}
$$

We define $\kappa_{T}=\alpha_{T} / \beta_{T}$ and find the relationship

$$
\kappa_{T}=\frac{\kappa}{1+\kappa \log \left(\Lambda z_{H}\right)} .
$$

Using this relationship, we can define the Kondo temperature in the holographic model by demanding that (26) diverges at this temperature for a given choice of $\kappa$ at some energy scale. It reads

$$
T_{\mathrm{K}}:=\frac{\Lambda}{2 \pi} e^{1 / \kappa(\Lambda)}
$$

and hence

$$
T / T_{\mathrm{K}}=\exp \left(-1 / \kappa_{T}\right)
$$

in the rescaled system. Note that $\kappa_{T}$ and the definition of $T_{\mathrm{K}}$ are invariant under $\mathrm{R} G$ translations $\Lambda \rightarrow \Lambda^{\prime}$, hence physical quantities. Once the coupling constant $\kappa$ is chosen at some energy scale $\Lambda$, a Kondo temperature can be defined for the model. That $\kappa$ indeed corresponds to the Kondo coupling constant on the field theory side is shown by holographic renormalisation in [78]. In this paper, we focus on the backreaction of the energy-momentum tensor on the brane. For this purpose, we do not need to renormalise our model since we do not compute any $n$-point functions or free energies.

To compute solutions for different ratios $\mu / T$, we increase $\mu_{T}$ and find that for $\mu_{T}>\mu_{T_{c}}$ the scalar field and thus $\alpha_{T}$ and $\beta_{T}$ become non-trivial and depend on $\mu_{T}$. On the field theory side this corresponds to the condensation of the scalar operator $\langle\mathcal{O}\rangle \neq 0$ below a critical temperature $T_{c}$. In the limit $\mu_{T} \rightarrow \mu_{T_{c}}$ the value of $\kappa_{T}$ converges to $\kappa_{T_{c}}$ and we can use 28 to define $T_{c}$ by

$$
T_{c}=T_{\mathrm{K}} \exp \left(-1 / \kappa_{T_{c}}\right) .
$$

Numerically we find $\kappa_{T_{c}} \approx 8.16$ and hence $T_{c} \approx 0.885 T_{\mathrm{K}}$, which is close to the values found in [48]. To summarise, there is a phase transition at a temperature $T_{c}$ which is approximately the Kondo temperature $T_{K}$. In this paper, we will not elaborate on this phase transition, which was described in more detail in [48], but we find the same qualitative features. 


\subsection{The normal phase}

As explained in the introduction, in the large $N$ model considered, the screened phase is characterised by a condensate of the operator $\mathcal{O}=\psi^{\dagger} \chi$ dual to the scalar field $\Phi$. We refer to the screened phase with $\langle\mathcal{O}\rangle \neq 0$ as the condensed phase, whereas we have $\langle\mathcal{O}\rangle=0$ in the normal phase. The UV fixed point is characterised by $\Phi=0, a_{m} \neq 0$. As discussed in [63], at the UV fixed point the energy-momentum tensor corresponds to that of a constant tension brane, with $S_{i j}=-\frac{\mathcal{N}}{2} \gamma_{i j} \mathcal{C}^{2}$ where the constant ${ }^{10}$ is given by $\mathcal{C}^{2}=-\frac{1}{2} f^{m n} f_{m n}$ and $\mathcal{C}$ is the electric flux of the YM field as described above. Such constant tension solutions have been studied before in thin-brane AdS/BCFT models [60 63, 95], and it is known that these solutions can be constructed by a geodesic normal flow, in an AdS or BTZ black hole background spacetime. This construction is obtained as follows. The starting point is the the trivial embedding $x_{+}(z)=0$ at the boundary. As $K_{\mu \nu}=0$ this embedding is totally geodesic, i.e. geodesics in the worldvolume of the brane are also geodesics with respect to the ambient spacetime. From every point on the brane, we construct a geodesic which is orthogonal to the brane at this point, which for our metric (7) can be done analytically. We follow each of these geodesics for a certain arclength $s>0$ and define a family of embeddings $X_{s}$ for each value of $s$. As explained in [63], each value of $s$ solves the Israel junction conditions for a specific constant tension on the brane. The gauge field on the brane is an explicit realisation of a constant tension solution if the scalar field vanishes. By solving the Israel junction conditions $(12)$, we find a relationship between the parameter $\mathcal{C}$ describing the gauge field solution and the parameter $s$ labelling the embedding, which is given by

$$
\tanh \left(\frac{s}{L}\right)=\frac{L}{4} \kappa_{N}^{2} \mathcal{N C}^{2}
$$

This construction is very useful: The spacelike geodesics which enter this normal flow construction coincide with those used for the Ryu-Takayanagi entanglement entropy prescription, if the entangling region is chosen to be an interval placed symmetrically around the impurity. For a constant tension brane, the results for the entanglement entropy hence take a very simple form. We will exploit this in section 3.3 below. Similar results may be found in 60 63, 95].

In the normal phase where $\langle\mathcal{O}\rangle=0$, the Israel junction conditions $(12)$ as well as the gauge field equations of motion (16) may be solved analytically. For $\mathcal{C}^{2}=-\frac{1}{2} f^{m n} f_{m n}$ and applying the radial gauge $a_{z}=0$, we find

$$
\begin{aligned}
a_{t} & =\frac{\mathcal{C} L^{2}}{z_{H}} \cosh (s / L)\left(\cosh (s / L)-\sqrt{\left(z_{H} / z\right)^{2}+\sinh ^{2}(s / L)}\right), \\
x_{+}(z) & =-z_{H} \operatorname{arctanh}\left(\frac{\sinh (s / L)}{\sqrt{\left(z_{H} / z\right)^{2}+\sinh ^{2}(s / L)}}\right) .
\end{aligned}
$$

It should be noted that for $\mathcal{C}^{2}>0$, the energy-momentum distribution on the brane described by (31), 32) satisfies the null and weak energy conditions (NEC and WEC) while violating the

\footnotetext{
${ }^{10}$ For an energy-momentum tensor of the form given here, the constancy of what is a priori the function $\mathcal{C}^{2}$ follows from the conservation of energy-momentum, $\nabla_{i} S^{i j}=0$.
} 
strong energy condition (SEC) which was for our 1+1-dimensional brane matter defined in 63 as

$$
\left(S_{i j}-S \gamma_{i j}\right) m^{i} m^{j} \geq 0 \text { for any timelike vector } m^{i} \text { tangent to the brane. }
$$

While the NEC enters the proof of the holographic $g$-theorem 60, 61], the violation of either WEC or SEC is required for our brane to reach the event horizon as in figure 2 instead of bending back to the boundary. This was shown in [63].

\section{Entanglement Entropy in the Holographic Model}

\subsection{Numerical results}

In this section we present the results that we obtain by solving the equations of motion (15)-(17) numerically. Moreover, we present our numerical results for the impurity entropy.

In the numerical calculations, the backreaction of the matter fields to the geometry is defined by fixing the ratio of the actions (6), (8) and (9) to $\kappa_{N}^{2} \mathcal{N}=1$. The electric flux $\mathcal{C}^{2}=-\left.\frac{1}{2} f^{m n} f_{m n}\right|_{z=0}$ of the gauge field $a$ at the conformal boundary is set to $\mathcal{C}=1 / 2$ as in [48], which fixes the leading behaviour of $Q$ in (21). Regularity at the horizon (20) fixes three more integration constants such that we are left with a one-parameter family of solutions, which we label by the value of the chemical potential $\mu_{T}=\mu / 2 \pi T$. Starting at the critical value $\mu_{T}\left(T_{c}\right)=\mu_{T_{c}}$, see below (21), we choose a set of increasing values of $\mu_{T}$. For each of those, we integrate the EOMs numerically from the boundary to the horizon. While doing this, we vary $\alpha_{T}$ and $\beta_{T}$ and until the regularity conditions (20) are satisfied. From this, we can compute the numerical embeddings at different ratios of $\mu / T$ or $T / T_{c}$, respectively. More details about the numerics can be found in appendix $\mathrm{B}$. It should be pointed out that many of the results that we present in this and the following sections are qualitatively constrained by very general properties of the system such as by the geometry of the BTZ black hole or by energy conditions [63]. Some of the results presented in section 3.3 will even be semi-analytical and applicable to more general models than our Kondo model. Hence, although we numerically fix $\kappa_{N}^{2} \mathcal{N}=1$ we expect that our results represent the qualitative features of our model for a general value of this parameter.

First of all, the numerical solutions for $x_{+}(z)$ (more specifically $\tilde{x}_{+}(\tilde{z})$, see $(25)$ ) are shown in figure 4. For $T / T_{c}=1$ we are in the normal phase, for which $x_{+}$is given by (32) which is the leftmost (red) curve in figure 4. As the temperature is lowered, the scalar $\phi$ condenses and hence adds a positive energy contribution (in the sense of the NEC) to the hypersurface energy-momentum tensor. As shown in [63], this leads to the embedding curves gradually bending more to the right, hence reducing the volume of the bulk spacetime. In fact, we found numerically that for all curves shown in figure 4 the NEC and WEC are satisfied while the SEC (as defined in (33)) is violated for every $z$. This means that $x_{+}^{\prime}(z)<0$ while $x_{+}^{\prime \prime}(z) \geq 0$ for every $z$, and that the brane indeed reaches the event horizon instead of turning around and returning to the boundary 63$]$. 


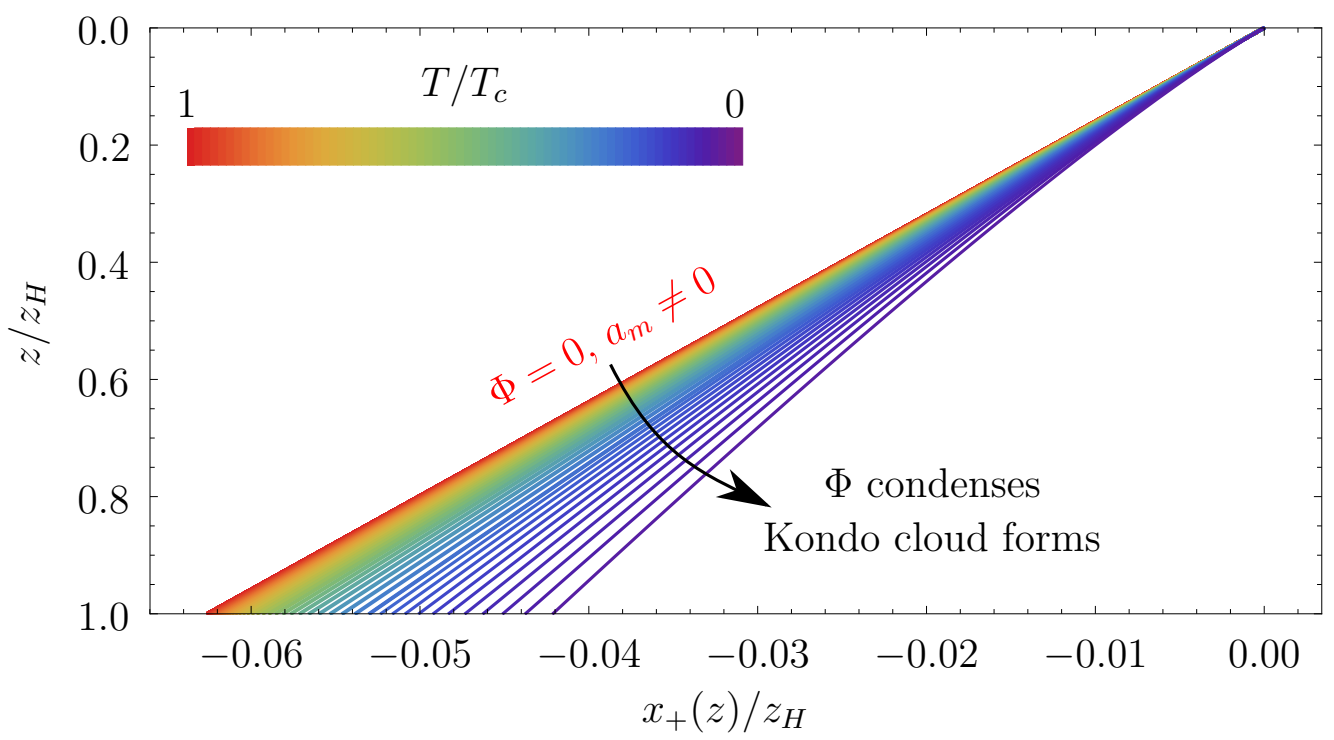

Figure 4: Embedding profiles $x_{+}(z)$ for different values of $T / T_{c}$, which is adjusted numerically by changing $\mu_{T}$, see below (25). The curves start out at the left for $T=T_{c}$ and bend to the right as $T / T_{c} \rightarrow 0$. Compare to figure 3 for the geometrical construction and the definition of $x_{+}(z)$. In particular, note that only the part of the spacetime to the right of the embedding curve is physical, while the left part is cut out and replaced by a mirror image of the right part.

We remind the reader that figure 4 only shows $x_{+}(z)$ and our full geometry is constructed as shown in figure 3 . This means that in figure 4 , for any given temperature the spacetime to the left of the brane is cut out, and the remaining part to the right of the brane is glued along the brane to its mirror image. Hence as the curves bend to the right in figure 4 for decreasing $T$, the (renormalised) volume of the spacetime (or spacial slices thereof) is also decreasing. This behaviour was already commented upon for constant tension models in [60 63, 95].

Let us now use these results to calculate the entanglement and impurity entropies numerically. Once the embedding functions $x_{+}(z)$ (and by symmetry assumption $x_{-}(z)=-x_{+}(z)$ ) are known, it is easy to holographically calculate entanglement entropies in the dual field theory using the RT prescription [58, 59]. This prescription states that for the $2+1$-dimensional background the entanglement entropy $S(A)$ of a boundary interval $A$ is given by

$$
S(A)=\frac{c}{6} \frac{\mathcal{L}_{A}}{L},
$$

where $\mathcal{L}_{A}$ is the length of a spacelike geodesic with its endpoints anchored to endpoints of the interval $A, c$ is the central charge of the boundary theory and we exploited the Brown-Henneaux formula $c=3 L / 2 G_{N}$.

To calculate the entanglement entropy, it is advantageous that by construction (see figure 3) the bulk spacetime is still given by the BTZ metric except for the brane itself. For BTZ, solutions to the geodesic equations are known analytically. On the field theory side, we aim at calculating 
the effect of the Kondo cloud on the entanglement and impurity entropies. For this purpose, we consider an interval of length $2 \ell$ that is symmetrically centered around the defect. As the defect is located at $x=0$ on the boundary, we consider intervals $(-\ell, \ell)$ on the $x$-axis ${ }^{11}$ This is shown in figure 2 ,

In parallel to the field theory discussion given in the introduction around (1), we define the impurity entropy also in the holographic context by

$$
\left.S_{i m p}(\ell) \equiv S(\ell)\right|_{\text {Impurity present }}-\left.S(\ell)\right|_{\text {Impurity absent }} .
$$

For the case without defect, we simply use the entanglement entropy in the BTZ background accoring to the RT prescription [58],

$$
\left.S(\ell)\right|_{\text {Impurity absent }}=S_{B H}(\ell)=\frac{c}{3} \log \left(\frac{1}{\pi \epsilon T} \sinh (2 \pi T \ell)\right) .
$$

The results for $S_{i m p}(\ell)$ as defined in (35) are shown in figure 5. The interpretation of these numerical results is straightforward. We see that in the normal phase, i.e. at $T / T_{c}=1$, we have $S_{i m p}(\ell)=$ const. as for this case the embedding function $\tilde{x}_{+}(\tilde{z})$ corresponds to an effective constant tension solution (32). Then as the temperature is lowered, the scalar field in the bulk becomes non-zero, which corresponds to the formation of the Kondo screening cloud. Due to the screening, the entanglement is reduced for fixed $\tilde{\ell}$ as $T / T_{c} \rightarrow 0$. From the bulk point of view, this is a consequence of following fact: Due to the NEC, the embedding curves $\tilde{x}_{+}(\tilde{z})$ increasingly bend to the right in figure 4 as $T / T_{c} \rightarrow 0$, and hence the bulk geodesics reaching the brane from the boundary point $\tilde{x}=+\tilde{\ell}$ have to travel a shorter distance, resulting in a reduced entanglement entropy. This can be interpreted as result of the Kondo cloud screening the impurity: The lower $T$ is for given $\tilde{\ell}$, the less impurity degrees of freedom are visible from outside the Kondo cloud. Near the boundary the scalar field falls off such that for small $\tilde{z}$ coordinates, the embedding curves $\tilde{x}_{+}(\tilde{z})$ deviate only slightly from the constant tension solution of the normal phase, as is shown in figure 4 . For this reason, for small $\tilde{\ell}$ the difference in entanglement entopy between condensed and normal phase goes to zero as displayed in figure 5 .

In figure 6 we take the results shown in figure 5, subtract the limit value $S_{\text {imp }}(\tilde{\ell} \rightarrow \infty)$ and present the resulting curves in a log-plot. The linear behaviour for large $\tilde{\ell}$ shows that our curves for $S_{i m p}(\tilde{\ell})$ display an exponential falloff $\sim$ const. $(T) \cdot e^{-2 \tilde{\ell}}$ towards the constant limiting value $S_{i m p}(\tilde{\ell} \rightarrow \infty)$. We see that while the exponent of the falloff behaviour is the same for all our curves, the prefactor changes as a function of $T$. This will be investigated more thoroughly and compared to the field theory results (3) in section 3.3 , yet the qualitative interpretation is clear: The larger the distance to the impurity is $(\tilde{\ell} \rightarrow \infty)$, the less prominent the influence of the impurity. This is due to the screening by the Kondo cloud.

It is tempting to relate the decrease of the volume of the bulk spacetime mentioned above to recent proposals concerning holographic measures of complexity, see $67-71$. The original

\footnotetext{
11 As we are interested in such symmetric intervals, the general refraction conditions for bulk geodesics at the brane reduce to the condition that the geodesics have to reach the brane at a right angle. See also 60 63, 95.
} 


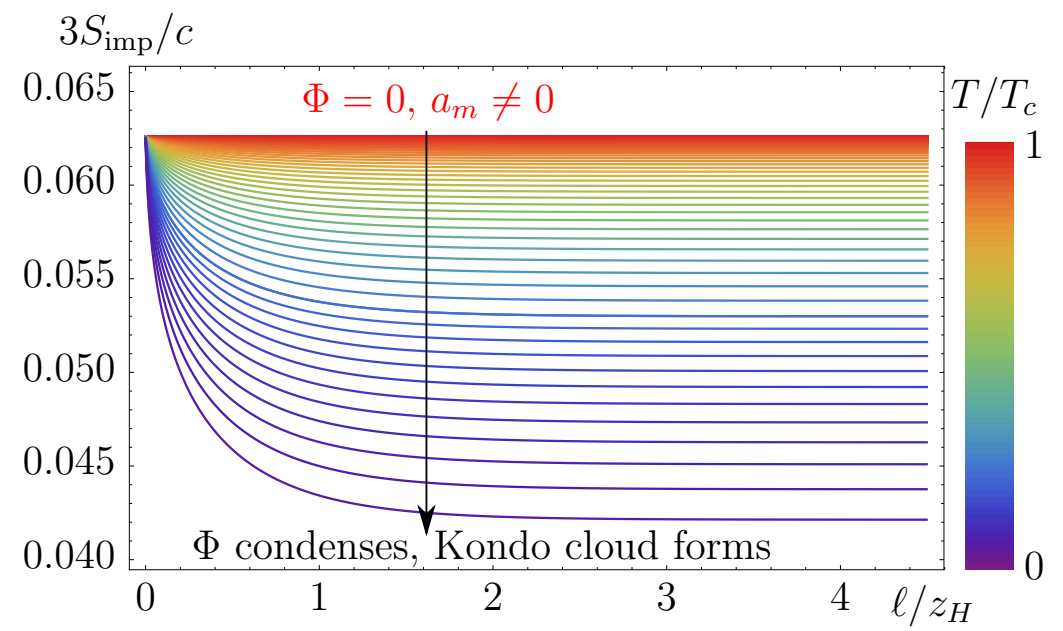

Figure 5: Numerical results for the impurity entropy from the holographic model. For $T / T_{c}=1$ the normal phase is given be a constant tension solution (32) that leads to a constant $S_{i m p}(\tilde{\ell})$ which is the uppermost curve in the figure. As the temperature is reduced and the scalar field condenses, due to the formation of the Kondo screening cloud the impurity entanglement for a given $\tilde{\ell}$ is reduced.

definition purported that holographically, a measure for the complexity $\mathfrak{C}$ of a quantum state can be defined as

$$
\mathfrak{C} \propto \frac{\mathcal{V}}{L G_{N}}
$$

where $\mathcal{V}$ is the volume of a codimension one spacelike hypersurface in the bulk geometry. In 70 this was generalised to the conjecture that $\mathfrak{C}$ can be obtained from the integral of the action over a certain bulk region. A thin brane construction with Israel junction conditions was employed in this context in [71]. We leave a detailed discussion of holographic complexity in our Kondo model for the future. Nevertheless, just as we will shortly interpret the decrease of entanglement entropy with the formation of the Kondo cloud as a sign of the screening of the impurity by that cloud, it is conceivable that the decrease of holographic complexity might be interpreted in the same way. In particular, our holographic map relates the decrease of entanglement to a reduction of the volume in the dual gravity theory.

\subsection{Holographic $g$-theorem}

Let us now investigate whether and how our holographic model obeys the $g$-theorem that is an analogue to the famous $c, a$ and $F$-theorems for boundary CFTs 22, 60, 64, 96. The $g$-theorem is certainly the most precise formulation of the idea that the Kondo cloud is a screening cloud: Along the RG flow from UV to IR, we expect the impurity entropy, i.e. the effective number of impurity degrees of freedom to decrease.

To do so, we first need to identify the boundary entropy from our numerical solutions, i.e. the

contribution of the boundary (or defect) to the entire system. In the standard AdS/CFT case 


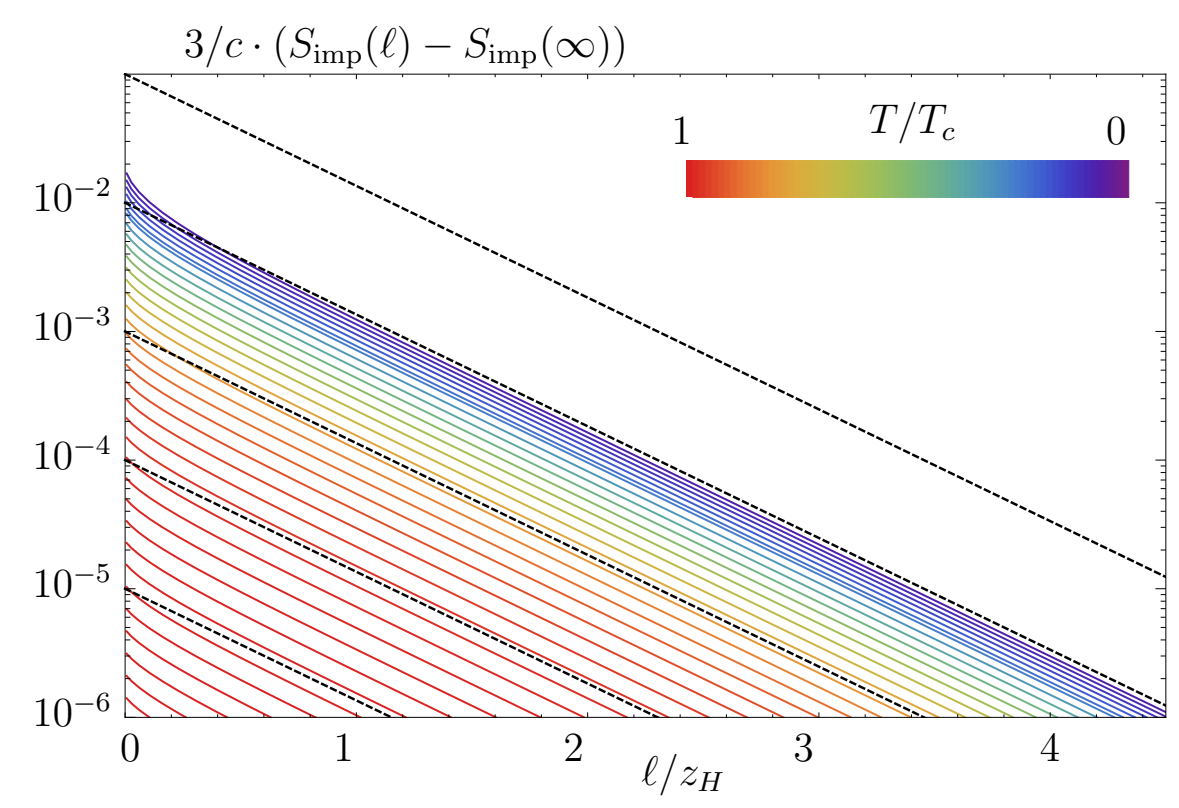

Figure 6: Exponential falloff of $S_{i m p}(\tilde{\ell})$ toward the limit value $S_{i m p}(\tilde{\ell} \rightarrow \infty)$ for large $\tilde{\ell}$. The temperature decreases from the bottom to the top curve. The dashed (black) lines show an exponential falloff of the form const. $(T) \cdot e^{-2 \tilde{\ell}}$ for comparison.

without defect, equation (36) defines the entanglement entropy for any interval of length $2 \ell$ on the boundary. As we take the limit $\ell \rightarrow \infty$ in which this interval spans all of the boundary, we enter a regime in which the entanglement entropy is linear in $\ell\left(S_{B H}(\ell) \sim \ell\right)$. In this regime, the entanglement entropy is not a good measure of quantum entanglement any more, but instead captures the thermodynamic entropy of the system which is of course expected to be an extensive quantity. From figure 5 we see that in this limit $\ell \rightarrow \infty$, the impurity entropy $S_{i m p}(\ell)$ approaches a constant limiting value which can be interpreted as the contribution of the defect to the thermodynamic entropy of the system. In fact, from the bulk geometry (see figure 4) we find that this additional entropy is just a result of the additional strip of the event horizon that we obtain because the brane is not located at the trivial embedding $x_{+}(z)=0$ any more. This trivial embedding corresponds to the absence of a defect. In particular 12

$$
S_{i m p}(\ell \rightarrow \infty)=\frac{c}{3} \cdot\left(-\tilde{x}_{+}\left(z_{H}\right)\right)
$$

where $\tilde{x}_{+} \equiv x_{+} / z_{H}$ as in (25). Using the temperature as energy scale of the RG-flow (and identifying $\left.\ln (g) \equiv S_{i m p}(\ell \rightarrow \infty)\right)$, it was shown in [64] that the $g$-theorem is equivalent to the requirement that the impurity entropy increases with temperature (or decreases with inverse temperature),

$$
T \cdot \frac{\partial S_{i m p}(\ell \rightarrow \infty)}{\partial T}>0
$$

12 Compared to $(34)$, we take $\mathcal{L}_{A}=-2 L \tilde{x}_{+}\left(z_{H}\right)$ since $\tilde{x}_{+}\left(z_{H}\right)$ is negative, and since we obtain two additional strips of event horizon, one to the left and one to the right of the brane, see figures 2 and 3 . The correct amount of the additional horizon area is then $-2 L \tilde{x}_{+}\left(z_{H}\right)$, not $-2 L x_{+}\left(z_{H}\right)$, due to the prefactor $\sim z^{-2}$ in the line element (7) evaluated at the horizon. 
along the RG flow, which is obviously the case in our example as seen from figures 5 and 7 . As explained in section 3.1, this is a consequence of the null energy condition (NEC): The more the scalar field condenses, the more it contributes positive energy (in the sense of NEC) to the brane, and hence the more the brane bends to the right in the IR in figure 4 as compared to the normal phase. This means $\left|\tilde{x}_{+}\left(z_{H}\right)\right|$ decreases as the temperature decreases, and with (38) equation (39) follows straightforwardly.

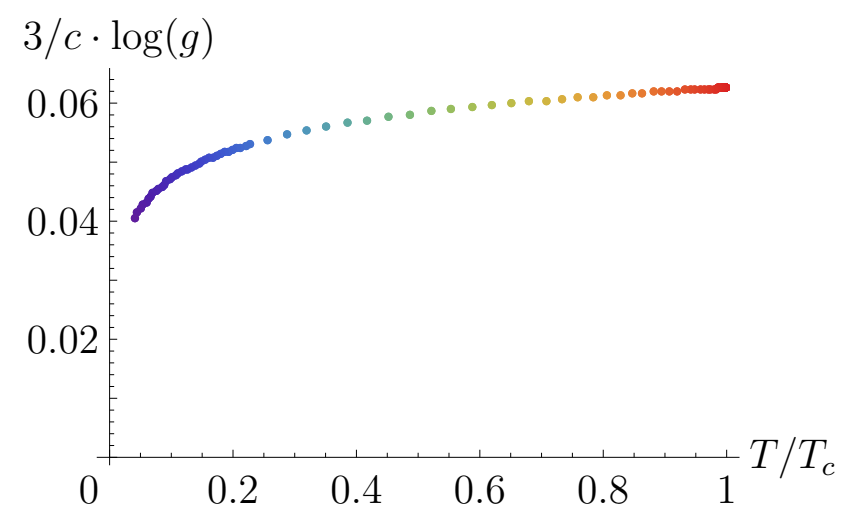

Figure 7: Boundary entropy $\ln (g)$ as a function of temperature.

In fact, the NEC already played a central role in the holographic proofs of the $g$-theorem that were given in [60, 96]. In [60], it was assumed that a thin brane described by (12) is embedded in a locally $\mathrm{AdS}$ spacetime as in the present paper, and the radial coordinate $z$ of the bulk spacetime was used as the scale of the RG flow. Let us use this interpretation here as well. Then, since the NEC is satisfied by the matter content of our holographic Kondo model, the $g$-theorem is guaranteed to hold in our case as well. As large $\ell$ on the boundary corresponds to large $z$ in the bulk, it is immediately obvious from figure 5 that $S_{i m p}(\equiv \ln (g)$ at conformal fixed points) decreases along the RG flow parametrised by $z$.

\subsection{Large $\ell$ approximation}

As our results for the brane embeddings are numerical, so far (in section 3.1) we only presented numerical results for the impurity entropy as well. Yet, as we show now, in the limit of large $\ell$ it is possible to derive the form of the impurity entropy $S_{i m p}$ due to very simple geometrical considerations.

The key to this is that both near the boundary and near the horizon, our brane may be approximated by a constant tension solution, i.e. a solution to equation 12 with $S_{i j} \equiv \frac{S}{2} \gamma_{i j}$. The reasons for this are simple: First, near the boundary the scalar field falls off $(\phi(z) \rightarrow 0)$, so that only the Yang-Mills field is left over, which describes a constant tension solution as explained in 63] and in section 2.3. In the case $\phi=0 \Leftrightarrow T / T_{c}=1$, this approximation becomes exact throughout the bulk. Second, near the event horizon a constant tension solution can always be fitted to the brane, in particular as due to the consistency conditions (20) and 
the vanishing of some components of $\widehat{\gamma}^{i j}$ at the horizon we find from (19) that the non-trace part of the energy-momentum tensor behaves as $S_{L / R} \rightarrow 0$ as $z \rightarrow z_{H}$. This constant tension brane then reaches the boundary at a shifted position $x=-D$ instead of $x=0$. This situation is shown in figure 8 .

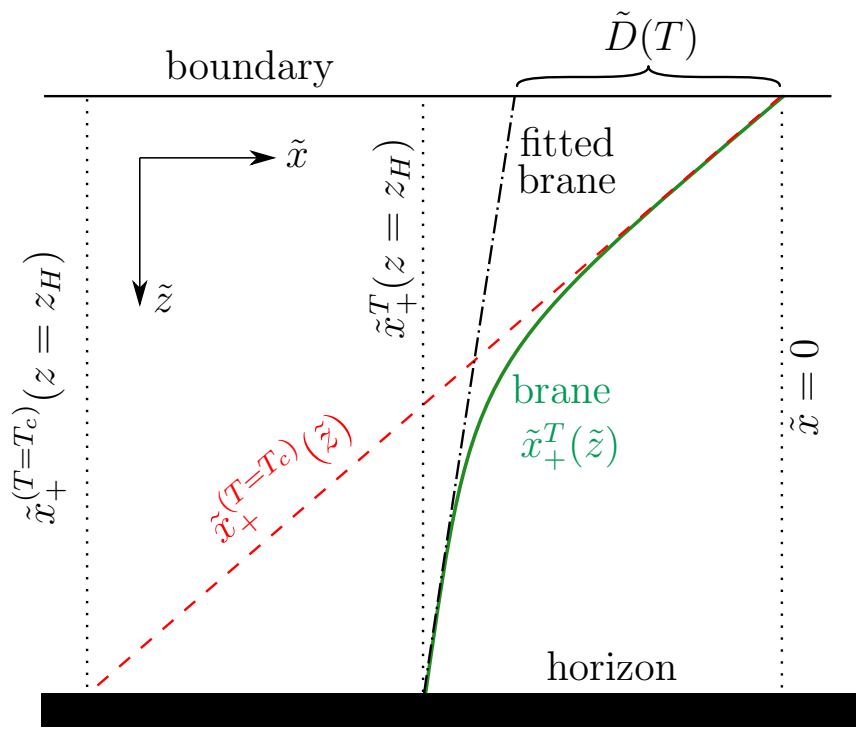

Figure 8: Sketch of the construction used for obtaining the impurity entropy in a linear approximation about the IR at finite temperature. Near the horizon, the brane is approximated by a constant tension solution. For the normal phase with $\phi=0$ or respectively at $T=T_{c}$, the brane is given by a constant tension solution (red dashed line). In contrast, for generic cases $T<T_{c}$ and $\phi \neq 0$, due to violation of SEC and compliance with NEC the brane bends to the right until it reaches the event horizon at $z=z_{H}$ (solid green line). Fitting a constant tension solution to this generic brane near the horizon (black dot-dashed line), we define the quantity $D(T)$ as the distance between the point where this fitted brane meets the boundary and the point $\tilde{x}=0$ where the original brane started from the boundary. Although the constant tension solutions are given by an arctanh formula (32), their curvature is not visible to the naked eye in the scale chosen for example in figure 4 so that they appear as straight lines.

As stated in section 2.3, the constant tension solutions can be constructed from a geodesic normal flow, using the same geodesics that are also used in the Ryu-Takayanagi prescription to calculate entanglement entropy for symmetric intervals around the impurity. Hence, if the standard result for the entanglement entropy of an interval of length $2 \ell$ in a BTZ background is (36), then the result for a constant tension brane will read, with tension $\lambda$ embedded in the BTZ background such that $x_{+}(0)=0$,

$$
S_{E E}^{\lambda}(\ell)=S_{B H}(\ell)+C(\lambda) .
$$

The larger $\ell$ is chosen to be, the deeper the entangling curves penetrate into the bulk, and accordingly the closer to the horizon they encounter the brane. Hence, the larger $\ell$ becomes, 
the more accurate it is to replace our brane by a constant tension brane with some tension $\lambda^{\prime}$ and anchored on the boundary at $x_{+}(z) \rightarrow-D$ as $z \rightarrow 0$, see figure 8 . In this approximation, the entanglement entropy reads

$$
S_{E E}^{\lambda^{\prime}, D}(\ell)=S_{B H}(\ell+D)+C\left(\lambda^{\prime}\right) .
$$

From this, (36) and the definition 35 it is easy to find

$$
\begin{aligned}
S_{i m p}(\ell) & =C\left(\lambda^{\prime}\right)+\frac{c}{3} \log \left(\frac{\sinh (2 \pi T(\ell+D))}{\sinh (2 \pi T \ell)}\right) \\
& \rightarrow C\left(\lambda^{\prime}\right)+\frac{c}{3}\left[2 \pi T D+\left(1-e^{-4 \pi T D}\right) e^{-4 \pi T \ell}+\mathcal{O}\left(e^{-8 \pi T \ell}\right)\right] \text { for } \ell \rightarrow \infty \\
& \sim C\left(\lambda^{\prime}\right)+\frac{c}{3}\left[2 \pi T D+4 \pi T D e^{-4 \pi T \ell}+\mathcal{O}\left(e^{-8 \pi T \ell}\right)\right] \text { for } \ell \rightarrow \infty \text { and small } D .
\end{aligned}
$$

Interestingly, if we assume $D$ to be small compared to both $\ell$ and $z_{H}$, we can simply approximate $S_{B H}(\ell+D)-S_{B H}(\ell) \sim D \cdot \partial_{\ell} S_{B H}(\ell)$ and hence

$$
S_{i m p}(\ell) \approx C\left(\lambda^{\prime}\right)+\frac{2 \pi c}{3} T D(T) \operatorname{coth}(2 \pi T \ell) \text { for } \ell T \gg 1 \text { and } D T \ll 1,
$$

where we reinstated the potential temperature dependence that $D$ may have on its own ${ }^{13}$ Using $-4 \pi T \ell=-2 \ell / z_{H}$, the formula (43) explains the exponential behaviour of our numerical results displayed in figure 6, while identifying the temperature dependent prefactor of the falloff with $1-e^{-4 \pi T D}$. Note that in contrast to equations (44) and (45), equation (43) does not require to assume that $D$ is small.

This holographic result may be compared directly to the field theory result (3) for $T \rightarrow 0, \ell \rightarrow$ $\infty$. First of all, the two expressions obviously differ in the constant boundary entropy term $C\left(\lambda^{\prime}\right)$, which itself may have a temperature dependence. It may in principle vanish as $T$ goes to zero. Second, the arguments of the coth agree exactly if we set $v=1$, i.e. if we assume that the Fermi velocity is equal to the speed of light. This is correct as we are working with massless chiral fermions in our model, see [48]. Finally, let us compare the prefactors of the coth in both (3) and (45). In the case of exact equality (and with $v=1$ ), these two equations imply for $T \approx 0$

$$
D(T) \approx \frac{\pi}{4} \xi_{\mathrm{K}} \Leftrightarrow \tilde{D}(T) \approx \frac{\pi^{2}}{2} \xi_{\mathrm{K}} T
$$

where we have used $2 \pi T D=D / z_{H}=\tilde{D}$ in accordance with (25). Of course, as we explained in the introduction, there are significant differences between the expected field theory in our holographic model and the field theory models used for deriving (3). Nevertheless, our results suggest that the simple geometric quantity $D(T)$, easily defined as shown in the sketch in figure 8, should approach a constant at low $T$. Equivalently, $\tilde{D}(T)$ which can be read off from figure

13 A similar argument can be made for the $T=0$ case. There we have $S_{A d S}(\ell)=\frac{c}{3} \log \left(\frac{2 \ell}{\epsilon}\right)$ instead of (36) and $D \cdot \partial_{\ell} S_{A d S}(\ell)=\frac{c D}{3 \ell}$ which can be compared to the $T=0$ result $(2)$. 
4 should show linear behaviour with $T$ for low temperatures. Then, $D(T=0)$ may be viewed as a measure for the Kondo scale $\xi_{\mathrm{K}}$ (up to a factor of order one). This is a very striking prediction.

Do our numerical results verify this claim (46)? In order to answer this question, we first have to discuss how much the temperature can rise before the behaviour (46) is lost. As it turns out, there is a very simple geometrical argument for this as we now explain. In fact, $\tilde{D}(T)$ is bounded from above. Thus for temperatures higher than $T=T^{*}$, the linear behaviour (46) is no longer possible.

The detailed argument for this is as follows. Due to the null energy condition (NEC), $\tilde{x}_{+}\left(z_{H}\right)$ of (25) can only increase along the boundary RG flow from the UV to the IR, i.e. $\left|\tilde{x}_{+}\left(z_{H}\right)\right|$ decreases. Moreover, due to the violation of the strong energy condition (SEC), we will always have $\tilde{x}_{+}^{\prime}\left(z_{H}\right)<0$. From this we derive that

$$
\tilde{D}(T) \leq\left|\tilde{x}_{+}^{(T)}\left(z_{H}\right)\right| \leq\left|\tilde{x}_{+}^{\left(T=T_{c}\right)}\left(z_{H}\right)\right| .
$$

This equation is easily understood from figure 8: The interval $\tilde{D}$ is shorter than the $\tilde{x}$-interval defined by the point where the brane solution (in solid green) meets the horizon. In turn, this interval is smaller than the $\tilde{x}$-interval defined by the point where the UV constant tension brane (in red, dashed) meets the horizon. As explained in section 3.2 , the quantity $\left|\tilde{x}_{+}^{\left(T=T_{c}\right)}\left(z_{H}\right)\right|$ in (47) has a very simple physical interpretation: It is half the length of the additional piece of event horizon that we obtain in the normal phase as compared to the BTZ black hole case without impurity (see footnote 12). In particular, as explained in the previous subsection it is related to the impurity entropy in the normal phase by

$$
\left|\tilde{x}_{+}^{\left(T=T_{c}\right)}\left(z_{H}\right)\right|=\frac{3}{c} \ln \left(g^{\left(T=T_{c}\right)}\right) .
$$

From $(32)$ and 30 , it follows that

$$
\left|\tilde{x}_{+}^{\left(T=T_{c}\right)}\left(z_{H}\right)\right|=s / L=\operatorname{arctanh}\left(\frac{L \kappa_{N}^{2} \mathcal{N} \mathcal{C}^{2}}{4}\right),
$$

where $\mathcal{C}$ here denotes the electric flux defined in section 2, In our numerics, we set $\kappa_{N}^{2} \mathcal{N}=1$, $\mathcal{C}=1 / 2$ and $L=1$, hence

$$
\left|\tilde{x}_{+}^{\left(T=T_{c}\right)}\left(z_{H}\right)\right|=\operatorname{arctanh}(1 / 16) \approx 0.0626,
$$

a number featuring prominently in figures 4,5 and 7 , This is now very important: $\tilde{D}(T)$ may initially grow linearly with $T$ as in (46), but if it is bounded from above by $\tilde{D}(T) \leq s / L$, this means that this linear behaviour has to stop (most likely long) before a temperature $T^{*}$ is reached where $\tilde{D}\left(T^{*}\right)=s / L$. The value of $T^{*}$ depends on the slope of the initial linear increase. If we assume for small $T / T_{c}$ that $\tilde{D}(T) \sim$ const. $\frac{T}{T_{c}}$, our estimate is that the linear behaviour of $\tilde{D}(T)$ can under no circumstances be expected to be seen above

$$
\frac{T}{T_{c}} \gtrsim \frac{T^{*}}{T_{c}} \approx 0.014
$$


were we have used (47) and by comparison with 46 we have set const. $=\pi^{2} T_{c} /\left(2 T_{\mathrm{K}}\right) \approx 4.37$. As this is only a bound, the linear behaviour may already end at a temperature orders of magnitude lower than this, see also figure 9 .

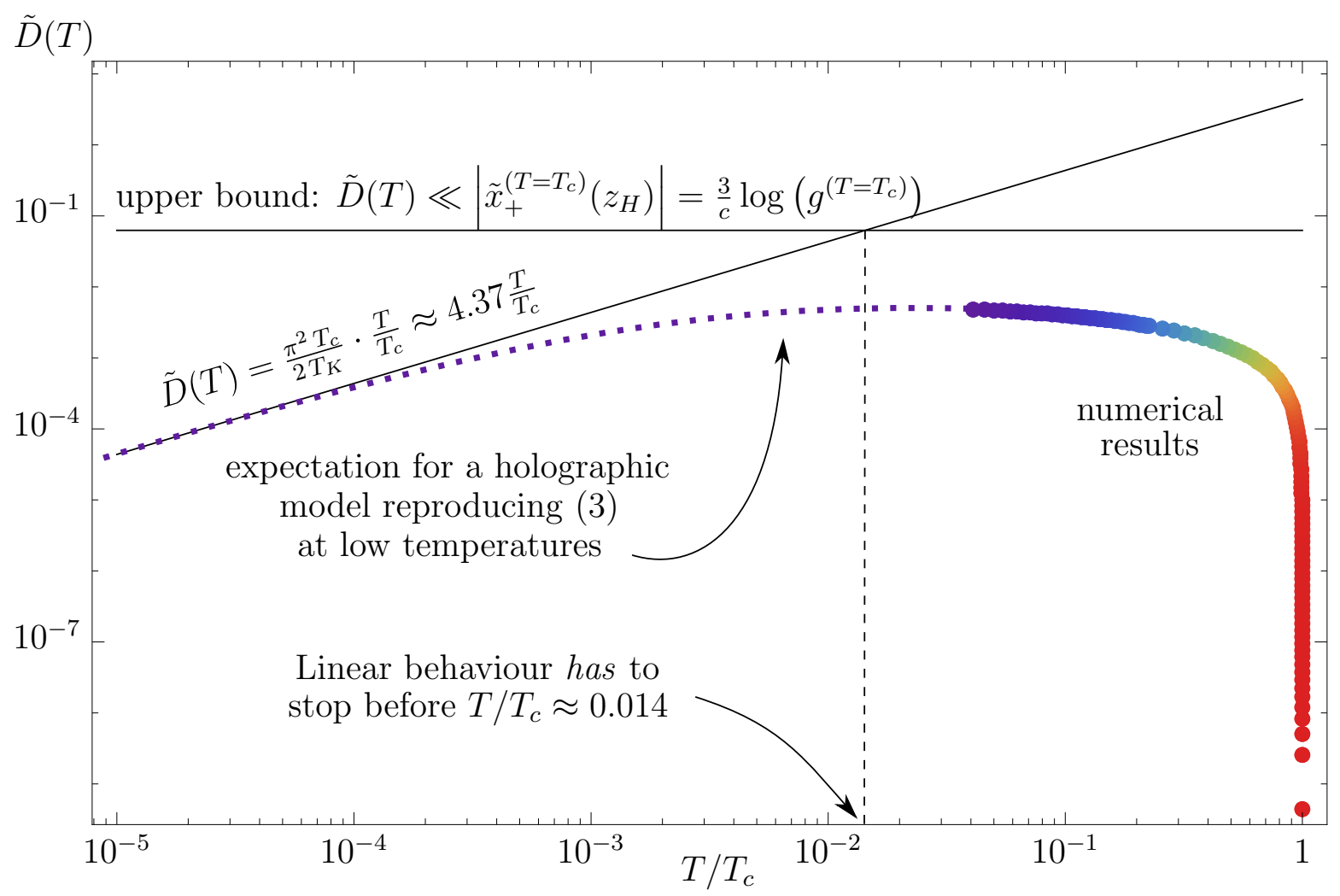

Figure 9: Numerical results for $\tilde{D}(T)=2 \pi T D(T)$ as a function of $T / T_{c}$ (large points), together with the expected behaviour of a holographic model reproducing (3) exactly at low $T$ (small points). As solid lines, we draw the upper bound on $D(T)$ given by 47 as well as a model of the form $\tilde{D}(T)=\frac{\pi^{2}}{2} \frac{T}{T_{\mathrm{K}}}$ which corresponds exactly to the field theory result $(3)$ as $T \rightarrow 0$.

To summarise this section, we have found that in certain limits (large $\ell$, small but finite $T$ ) we may qualitatively reproduce field theory results for the impurity entropy from simple geometrical considerations. This applies in particular to the exponential falloff following from the field theory result (3). These arguments are analytical and only dependent on general geometric properties of our model (such as the BTZ background geometry that implies (36)). This indicates that our approach of approximating the curved brane of our holographic model by a constant tension brane near the event horizon (figure 8) corresponds to a linear perturbation about the IR fixed point. For this reason, we expect the results (43) and, where the necessary approximations are valid, also (45) to be examples for universal behaviour of holographic models of $\mathrm{AdS}_{3} / \mathrm{BCFT}_{2}$ duality involving thin branes [60 62], beyond the specific Kondo model studied here. Furthermore, it became apparent that while the coth terms in (3), (45) agreed exactly in their respective arguments, we did not find even qualitative agreement of the prefactors of the coth term, as far as their dependence on temperature is concerned, see figure 9. In 
fact, we ended the section by showing that even if there were an agreement of these prefactors, it could only be manifest at very low temperatures, lower than what we were able to study numerically. We believe that the argument we used to show this is interesting in its own right: While the equations (3), 45) were clearly derived under the assumption of low temperature $\left(T / T_{c} \ll 1\right)$, we did not specify what "low" means quantitatively in this context. However, in (51) we derived a bound on this low temperature behaviour. Curiously, this bound was derived from equations (47) and (48), which are dependent on the high temperature $\left(T=T_{c}\right)$ properties of our system.

Based on these findings and especially on the results displayed in figure 9, it would certainly be interesting to study our system over several orders of magnitude of temperatue. However, not only has it turned out to be very demanding to extend our numerical results to low values of $T / T_{c}$ with adequate accuracy: As we show in the next section, a physical behaviour at low temperatures can only be expected for more complicated forms of the potential $V\left(\Phi^{\dagger} \Phi\right)$ than the square term (14) used so far.

\section{$3.4 T=0$ behaviour}

As pointed out in the previous section, it is interesting for us how our system behaves as we approach $T=0$. This could be studied directly by embedding our brane into a Poincaré background instead of a BTZ metric as before, but unfortunately this is very demanding to do numerically. As we show in this section, we may extract some information about this case by using analytical arguments. The zero temperature limit of holographic superconductors was studied for instance in [97].

The main question of the $T=0$ case is: Will our system flow to a conformal fixed point? In our AdS/BCFT like ansatz, this would mean that at $T=0$ our system approaches a constant tension solution, at least deep in the bulk 60 62. We would thus expect

$$
\begin{aligned}
S_{i j} & \rightarrow \text { const. } \cdot \gamma_{i j} \text { as } z \rightarrow \infty \\
\Rightarrow S_{L / R} & \rightarrow 0 \text { as } z \rightarrow \infty
\end{aligned}
$$

for the energy-momentum tensor and components defined in (11) and (19), if indeed we find

a conformal IR fixed point. From the specific form of both $S_{L / R}$ and $\mathcal{K}_{L / R}$ (see equation (19) and [63]), this translates to the set of assumptions

$$
S_{L / R} \rightarrow 0, \phi \rightarrow \text { const. } \neq 0, a_{t} \rightarrow 0, x^{\prime}(z) \rightarrow \text { const., as } z \rightarrow \infty
$$

where, as $\widehat{\gamma}^{i j}$ behaves as $\sim z^{2}$, we can additionally show that $x^{\prime \prime}(z)$, $\phi^{\prime}$ and $a_{t}$ have to approach zero faster than $z^{-1}$. This has to be contrasted with the scalar equation of motion

$$
\partial_{m}\left(\sqrt{-\gamma} \gamma^{m n} \partial_{n} \phi\right)=\sqrt{-\gamma} \gamma^{m n} a_{n} a_{m} \phi+\frac{1}{2} \sqrt{-\gamma} \frac{\partial V}{\partial \phi}
$$

where we used the same gauge choice as in (15)-(17). Suppose that as $\phi \rightarrow$ const. $\neq 0$ the term $\frac{\partial V}{\partial \phi} \neq 0$. For our simple quadratic potential this is inevitable, but here we want to investigate 
the more general case. Due to the factor $\sqrt{-\gamma}$, the last term in (55) will then go to zero as $z^{-2}$ for $z \rightarrow \infty$. This means that (55) can only be satisfied if any other of the terms in this equation behaves exactly as $z^{-2}$ for large $z$, too. Unfortunately, from (54) and our reasoning below this equation, we can actually deduce that all the other terms in (55) go to zero strictly faster than $z^{-2}$ for large $z$. This contradiction leads to two conclusions: First, for our simple model with potential $V \sim \phi^{2}$ the equations of motion seem to forbid the existence of an IR fixed point in our model. Second, for an IR fixed point to exist, it is necessary that for large $z \phi \rightarrow \phi^{*}$ such that $\left.\frac{\partial V}{\partial \phi}\right|_{\phi=\phi^{*}}=0$. This can easily be achieved by adding quartic or higher terms to the potential of our model. We then expect that our model with square potential is a good approximation to this improved model only when the maximal value of $\phi(z)$ is small compared to $\phi^{*}$. Differences between these two models would then only become apparent at low temperatures when the scalar field $\phi$ grows in the bulk so that the higher order terms in the potential become important. For now, we leave the study of a model with a more complicated potential for future research and continue to summarise the results obtained with our original simple holographic Kondo model as studied in [48,63].

\section{Conclusions and Outlook}

\subsection{Conclusion}

Let us recapitulate the main results of this paper. In section 3.1 we have presented numerical results on the impurity entropy in the holographic model of the Kondo effect that was studied in [48, 63 as summarised in section 2. We discussed how several qualitative features resulted straightforwardly from arguments concerning the bulk geometry based on the energy conditions that are satisfied or violated by the matter-content of the bulk model. In particular, we found a qualitative geometrical dual gravity picture demonstrating how the Kondo cloud acts as a screening cloud hiding the impurity. From the field theory side it is expected [22] that due to the boundary RG flow, the impurity effectively looses degrees of freedom along the RG flow from the UV to the IR. This corresponds to the screening. In our holographic model, there are three clear properties that we interpret to correspond to this: First, the flux of the Yang-Mills field $a$ is decreasing when moving along the brane from the UV to the IR. This was studied in detail in [48] for the case without backreaction and qualitatively carries over to our numerical solutions as well. Second, we find that due to the null energy condition (NEC) the renormalised volume of our bulk spacetime is reduced for decreasing temperature, a feature that may be interpreted as a reduced holographic complexity, see $67-71$. Third, the reduction of the impurity entropy both with decreasing $T$ and increasing $\ell$ is a clear sign of the screening of the impurity.

This was formalised in section 3.2 in form of the holographic $g$-theorem 22 , 64 that has attracted interest in holographic research before 60 62, 96]. Due to the proof given in [60], it came as no surprise that we found the $g$-theorem to be satisfied by our explicit example, specifically as a consequence of the NEC being satisfied by the matter fields in our holographic model. 
In section 3.3 we derived an analytical approximation formula to our numerical results valid for large entanglement interval sizes $\ell$. This made it possible to compare our results to similar field theory results that are summarised in the introduction, with remarkable agreement. Indeed, the generality of the construction employed in section 3.3 led us to view this as a geometrical bulk manifestation of the universality of perturbing about an IR fixed point, at least to first order. For systems that can be described by a constant tension (bulk) solution at $T=0$ in the AdS/BCFT approach [60 62], we found that an impurity length scale $\left(\frac{\pi}{4} \xi_{\mathrm{K}}\right.$ in the case of our Kondo model) can be identified with a geometrical bulk length $D$ as defined in figure 8 . In comparing gravity and field theory results, the significance of the small-temperature behaviour of our model became apparent.

\subsection{Outlook}

There are many open questions related to impurities in strongly coupled systems. The results of the present paper, which provide a new link between the impurity entropy and defects in spacetime geometry, may be applied in a number of different scenarios. In the immediate holographic context, we note the papers [98,99], where vortices in holographic superfluids were considered as defects in a 2+1-dimensional CFT. Also in that case, the theory flows to a nontrivial fixed point in the IR: At low energies, the vortex corresponds to a conformal defect interacting with the gapless modes in the superfluid.

For Kondo or similar impurity systems, entanglement entropy is not the only measure of entanglement, others such as Rényi entropies and entanglement negativity (to only name a few) have been studied in 65, 100 102]. How to study these measures of entanglement in our holographic Kondo model is an interesting task that we leave to the future. The results of [101] for the twoimpurity Kondo model are especially interesting in the light of the recent generalisation [78] of the holographic Kondo model [48] to the two-impurity case. Following the direction of research begun in [78], it would of course also be interesting to study spatially separated impurities and Kondo lattices. Moreover, it will also be interesting to analyse the case of zero temperature. This may be compared to condensed matter results, as for instance in $27,30,103$.

Moreover, the Kondo effect was recently discussed in the context of QCD [104], where it was found to occur in light quark matter which contains heavy quarks as impurities. It will be illuminating to compare the QCD Kondo model with the holographic one discussed here.

In view of the new recent interaction between gauge/gravity duality and quantum information theory, we note that the Kondo model was studied in the MERA approach for instance in [105]. Here our model, which links the removal of points from the geometry to the decrease of information, may also prove useful. Indeed in sections 3.1 and 4.1 we pointed out that the screening of the impurity by the Kondo cloud might manifest itself not only in the entanglement or impurity entropy, but also in a quantity referred to as holographic complexity [67 71$]$. It will certainly be interesting to study this in more detail, calculating the holographic (impurity-) complexity numerically and investigating its behaviour under the boundary RG flow. One interesting question might be whether the holographic $g$-theorem can be phrased in terms of 
complexity instead of entanglement entropy.

\section{Acknowledgements}

We are very grateful to Ian Affleck, John Estes, Nabil Iqbal, Kristan Jensen, Henrik Johannesson, Charles Melby-Thompson, Thore Posske, Subir Sachdev, Pasquale Sodano, Ioannis Papadimitriou, Jonas Probst and Wolfgang Mück for illuminating discussions. We are particularly grateful to Andy O'Bannon for extremely useful comments. JE, MF and CH thank the Galileo Galilei Institute for Theoretical Physics for the hospitality and the INFN for partial support during the course of this work. JE, MF and JMSW are grateful to the IPMU Tokyo for hospitality. JMSW thanks NCTS for support during the course of this work. This work is partially supported by the Spanish grant MINECO-13-FPA2012-35043-C02-02. CH is supported by the Ramon y Cajal fellowship RYC-2012-10370. JE thanks the KITPC Beijing and the IIP Natal for hospitality. Moreover, she thanks the participants of the school 'Strongly coupled field theories for condensed matter and quantum information theory' in Natal for discussions.

\section{A Junction Conditions for Abelian Chern-Simons Fields}

Here, we derive the junction conditions that govern the interaction of our (abelian) bulk ChernSimons field with the brane worldvolume current. As for the Israel junction conditions (12) of the gravitational field, this can be done in different ways. We consider the case that the current is smoothed out as described below, such that after taking a well-defined limit it becomes a delta distribution. For a related study of the behaviour of Chern-Simons fields in the presence of domain walls, see [106, 107.

Later on, we will phrase our junction conditions in a manifestly gauge and coordinate invariant way, such that our results can be readily applied to the Kondo model. However, for now assume that we work in a Gaussian normal coordinate system, such that the brane is located at $x=0$. In our Kondo model, the current involving the charged scalar $\Phi=\phi e^{i \psi}$ on the brane worldvolume takes the form

$$
J^{m}=\gamma^{m n} i\left(\Phi \mathcal{D}_{n} \Phi^{\dagger}-\Phi^{\dagger} \mathcal{D}_{n} \Phi\right)=2 \gamma^{m n}\left(A_{n}-a_{n}+\partial_{n} \psi\right) \phi^{2},
$$

which in the static case can be written as $J^{m} \sim \mathcal{Q}(z)\left(\partial_{t}\right)^{m}$ in radial gauge. The brane is now assumed to be smoothed out by a profile satisfying $f(x)=F^{\prime}(x)$, such that there exists a limit in which $f(x) \rightarrow \delta(x), F(x) \rightarrow \theta(x) \stackrel{14}{\square}$ See figure 10 for the setup.

As we assume a static situation, all objects are independent of the coordinate $t$. With the CS equation of motion

$$
\epsilon^{\rho \mu \nu} F_{\mu \nu}=-4 \pi J^{\rho}
$$

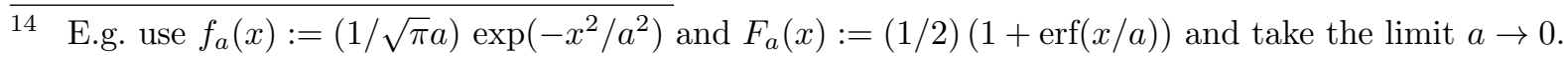




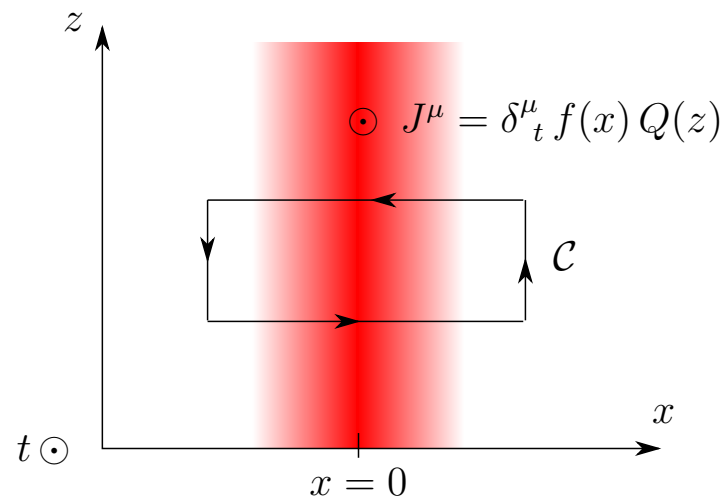

Figure 10: A static worldvolume current of the brane at $x=0$, smoothed out by a profile $f(x) . \mathcal{C}$ is the path of a possible Wilson loop.

the only non-trivial equation of motion then reads

$$
2 \epsilon^{t z}\left(\partial_{z} A_{x}-\partial_{x} A_{z}\right)=-4 \pi J^{t}=-4 \pi f(x) \mathcal{Q}(z),
$$

where we define the worldvolume $\epsilon$-tensor by contracting the bulk $\epsilon$-tensor with the normal form of the brane. In Gaussian normal coordinates, this simply means $\epsilon^{\rho \mu x} \equiv \epsilon^{\rho \mu}$. Equation (58) can be solved in two ways:

Gauge $A_{x}=0:$ First we assume $A_{x}=0$, hence

$$
\begin{aligned}
-\epsilon^{t z} \partial_{x} A_{z} & =-2 \pi f(x) \mathcal{Q}(z), \\
\Rightarrow \epsilon^{t z} A_{z} & =2 \pi F(x) \mathcal{Q}(z) .
\end{aligned}
$$

In the limit $F \rightarrow \Theta, f \rightarrow \delta$ this means that the parallel component of the CS field has a discontinuity proportional to the charge density $\mathcal{Q}$ on the brane.

Gauge $A_{z}^{\prime}=0:$ If we assume $A_{z}^{\prime}=0$ instead, we find

$$
\begin{aligned}
\epsilon^{t z} \partial_{z} A_{x}^{\prime} & =-2 \pi f(x) \mathcal{Q}(z) \\
\Rightarrow-\tilde{\epsilon}^{t z} A_{x}^{\prime} & =2 \pi f(x) \int_{0}^{z} \sqrt{-\gamma} \mathcal{Q}(\hat{z}) \mathrm{d} \hat{z},
\end{aligned}
$$

where $\tilde{\epsilon}$ denotes the Levi-Civita symbol, which is related to the Levi-Civita tensor by $\epsilon^{m n}=$ $\tilde{\epsilon}^{m n} / \sqrt{-\gamma(z)}$. In the limit $F \rightarrow \Theta, f \rightarrow \delta$ we see that in this gauge, the component of the Chern-Simons field normal to the brane acquires a contribution $\sim \delta(x)$. 
Both approaches are indeed gauge-equivalent. This may be seen by explicitly showing that the difference of both solutions (60) and $(62)$ is a total derivative $\mathrm{d} \alpha=A^{\prime}-A$ with

$$
\alpha=\frac{2 \pi}{\tilde{\epsilon}^{t z}} F(x) \int_{0}^{z} \sqrt{-\gamma} \mathcal{Q}(\hat{z}) \mathrm{d} \hat{z} .
$$

In the limit in which the current distribution becomes infinitely thin, this means that the gauge function $\alpha$ approaches the Heaviside $\theta$ function, and hence $\alpha$ need not vanish for large $|x|$. Moreover, Wilson lines transform in the appropriate way

$$
W(a, b) \rightarrow e^{i \alpha(a)} W(a, b) e^{-i \alpha(b)} .
$$

For a simple rectangular path such as $\mathcal{C}$ in figure 10, we may explicitly calculate the Wilson loop in the two gauges presented above and check that the results agree. By the equations of motion of the CS field, we find

$$
\oint_{\mathcal{C}} A=\int_{\operatorname{int}(\mathcal{C})} d A \stackrel{E O M s}{\propto} \int_{\operatorname{int}(\mathcal{C})} \mathcal{Q},
$$

where $\operatorname{int}(\mathcal{C})$ denotes the interiour of $\mathcal{C}$.

What we have learned above is that away from the brane on which the current $J^{\mu}$ is localised, the CS field satisfies its bulk (vacuum) equations of motion, while at the brane, depending on a gauge choice, we either have a discontinuity in the parallel component of the $A$-field or a $\delta$-peak in the normal component. In the former case, the projection of the CS field to the brane worldvolume is not uniquely defined. For symmetry reasons, we assume that in equations (10), (56) the scalar field $\Phi$ couples to the mean value of the CS fields projected onto the brane from the left (with projector $\overrightarrow{\mathcal{P}}$ ) and from the right (with projector $\overleftarrow{\mathcal{P}}$ ) side of the bulk:

$$
A_{m} \equiv \frac{1}{2}\left(\overleftarrow{\mathcal{P}}_{m}^{\mu} A_{\mu}+\overrightarrow{\mathcal{P}}_{m}{ }^{\mu} A_{\mu}\right)
$$

In addition, we define the discontinuity of the parallel components at the brane by

$$
C_{m} \equiv \overleftarrow{\mathcal{P}}_{m}{ }^{\mu} A_{\mu}-\overrightarrow{\mathcal{P}}_{m}{ }^{\mu} A_{\mu}
$$

and the $\delta$-contribution to the normal component of the CS field by

$$
A^{0}(z, t) \equiv \int_{-\epsilon}^{\epsilon} A^{\mu} n_{\mu} \mathrm{d} s
$$

where $n_{\mu}$ is the normal form of the brane and the integral is along an integral curve of the vector field $n^{\mu}$ that crosses the brane at coordinates $(z, t)$. Taking the above results and definitions 
together, we then find that at the location of the brane, the CS field satisfies the junction condition

$$
-2 \pi J^{m}=\epsilon^{i m}\left(C_{i}-\partial_{i} A^{0}\right)
$$

Let us comment on the interesting properties of this equation. First of all, we note that in the equations of motion of the scalar field and the Yang-Mills field, as well as in the current (56), only the projection $A_{m}$ as defined in (66) plays a role, while the quantities $C_{m}$ and $A^{0}$ appear only on the right hand side of (69). Furthermore, for given $J^{m}$ equation (69) is an algebraic equation for $C_{m}$ and $A^{0}$. This means that just as in 48 the CS field decouples from the dynamics of the brane and we can first gauge $A_{m}=0$ in our setup, then solve for $\Phi, a_{m}$ and $J^{m}$ and then solve the algebraic equation (69). Finally, it is interesting to note the form of 69 in which $J^{m}$ and hence the combination $\left(C_{i}-\partial_{i} A^{0}\right)$ are gauge invariant by definition, such that the quantity $A^{0}$ acts as a gauge function for $C_{m}$.

\section{B Details of Numerics}

Here we briefly describe our method to solve the equations of motion (15)-(17) numerically. First of all we exploit two scaling symmetries of the EOMs to set $L=1$ and $z_{H}=1$. All coordinates become dimensionless quantities: $\tilde{z}=z / z_{H}, \tilde{x}=x / z_{H}, \tilde{t}=t / z_{H}$. The relationship between the matter action and the gravity action is fixed by $\kappa_{N}^{2} \mathcal{N}=1$. The electric flux defined in section 2.2 is set to $\mathcal{C}=1 / 2$ to be consistent with [48].

The $\tilde{z} \tilde{z}$-component of (17) only contains first derivatives of the embedding function $\tilde{x}_{+}(\tilde{z})$. It can be analytically solved for $\tilde{x}_{+}^{\prime}(\tilde{z})$ as a function of $\phi$ and $a_{t}$ as well as their derivatives. There are four solutions to this equation. However, only one of them is consistent with the background solution (32). We take this one and insert it into the equations of motion for $\phi$ and $a_{t}$. These are now two coupled second order ODEs for two fields and hence require four boundary conditions.

To solve them numerically, we apply the shooting method. We expand the fields at the asymptotic boundary $\left(\tilde{z}=0\right.$, UV) and at the horizon $\left(\tilde{z}=1\right.$, i.e. $z=z_{H}$, IR). Since the mass of the scalar is fine-tuned to saturate the Breitenlohner-Freedman bound (22), the UV expansions come along with roughly $\mathcal{O}(1)+3 k$ logarithms at $k^{\text {th }}$ order in $\tilde{z}$ :

$$
\begin{aligned}
& \phi(\tilde{z}) \sim \sqrt{\tilde{z}}\left(\alpha_{T} \log (\tilde{z})+\beta_{T}+\sum_{k=1}^{\infty} \sum_{j=0}^{3 k+3}\left(c_{\phi}(k, j) \tilde{z}^{k} \log (\tilde{z})^{j}\right)\right) \\
& a_{t}(\tilde{z}) \sim Q \frac{z_{H}}{\tilde{z}}+\mu_{T}+\sum_{k=1}^{\infty} \sum_{j=0}^{3 k+4}\left(c_{a}(k, j) \tilde{z}^{k} \log (\tilde{z})^{j}\right)
\end{aligned}
$$

For this reason, expanding the fields in the UV using MATHEMATICA takes remarkably long and we were able to expand the fields only up to $4^{\text {th }}$ order in the UV. In the IR, we expand 
to $8^{\text {th }}$ order. $Q$ is fixed by 21 and our choice of $\kappa_{N}^{2} \mathcal{N}$ and $\mathcal{C}$. Regularity at the horizon fixes two more integration constants such that we are left with a one-parameter family of solutions, which we label by the value of the chemical potential $\mu_{T}=\mu / 2 \pi T_{c}$. Starting at the critical value $\mu_{T}=\mu_{T_{c}}$, see below (21), we choose a set of increasing values of $\mu_{T}$. For each of those we vary $\alpha_{T}$ and $\beta_{T}$ and integrate the EOMs numerically from the boundary to the horizon until the regularity conditions (20) are satisfied. Hence, each solution can also be referred to by $\kappa_{T}$ and, by (28) and (29), a value of $T / T_{c}$.

Next, we define a set of radial positions between the conformal boundary and the horizon. For each position we obtain the embedding profiles $\tilde{x}_{+}(\tilde{z})$ by integrating numerically $\tilde{x}_{+}^{\prime}(\tilde{z})$ as a function of $\phi$ and $a_{t}$, using the numerical solutions found by the shooting. We choose the integration constant such that $\tilde{x}_{+}(0)=0$, which is possible due to translational symmetry of the BTZ metric (7).

We obtain numerical data in the form of $\left(\tilde{z}, \tilde{x}_{+}(\tilde{z}), \tilde{x}_{+}^{\prime}(\tilde{z})\right)$ for different $\tilde{z}$, which can be mapped to a unique geodesic starting normal to the brane at $\left(\tilde{z}, \tilde{x}_{+}(\tilde{z})\right)$ and ending at the asymptotic boundary at $\tilde{z}=0$ and $\tilde{x}=\tilde{\ell}$. The geodesics are regularised by subtracting the divergent term $\log (2 / \tilde{z})$ as we take the limit $\tilde{z} \rightarrow 0$ and have regularised (dimensionless) length $\mathcal{L}(\tilde{\ell}) / L$, where we reintroduced the AdS radius $L$ appearing in the line element.

Thus, starting at one position $\left(\tilde{z}, \tilde{x}_{+}(\tilde{z})\right)$ on the brane, we obtain data of the form $(\mathcal{L}(\tilde{\ell}) / L, \tilde{\ell})$. Repeating this procedure for many starting points on the brane and for different $\mu_{T}$, i.e. $T / T_{c}$, we can interpolate a function $\mathcal{L}(\tilde{\ell}) / L$. Combined with (34), we obtain the entanglement entropy as a function of the subinterval around the impurity and the temperature. This is how we compute the first term in the definition of the impurity entropy (35). The second term is known analytically $(36)$.

\section{References}

[1] J. Kondo, "Resistance Minimum in Dilute Magnetic Alloys," Progress of Theoretical Physics 32 no. 1, (1964) 37-49.

[2] K. G. Wilson, "The Renormalization Group: Critical Phenomena and the Kondo Problem," Rev.Mod.Phys. 47 (1975) 773.

[3] H. R. Krishna-murthy, J. W. Wilkins, and K. G. Wilson, "Renormalization-group approach to the Anderson model of dilute magnetic alloys. I. Static properties for the symmetric case," Phys. Rev. B 21 (Feb, 1980) 1003-1043.

[4] H. R. Krishna-murthy, J. W. Wilkins, and K. G. Wilson, "Renormalization-group approach to the Anderson model of dilute magnetic alloys. II. Static properties for the asymmetric case," Phys. Rev. B 21 (Feb, 1980) 1044-1083.

[5] N. Andrei, "Diagonalization of the Kondo Hamiltonian," Phys. Rev. Lett. 45 (Aug, 1980) 379-382. 
[6] P. Wiegmann, "Exact Solution of s-d Exchange Model at T=0," Sov. Phys. JETP Lett. 31 (1980) 364.

[7] N. Andrei, K. Furuya, and J. H. Lowenstein, "Solution of the Kondo problem," Rev. Mod. Phys. 55 (Apr, 1983) 331-402.

[8] A. Tsvelick and P. Wiegmann, "Exact Results in the Theory of Magnetic Alloys," Advances in Physics 32 no. 4, (1983) 453-713.

[9] P. Coleman and N. Andrei, "Diagonalisation of the Generalised Anderson Model," Jour. Phys. C19 (1986) 3211-3233.

[10] N. Andrei, "Integrble Models in Condensed Matter Physics," eprint arXiv:cond-mat/9408101 (Aug., 1994), cond-mat/9408101.

[11] P. Zinn-Justin and N. Andrei, "The Generalized Multi-channel Kondo Model: Thermodynamics and Fusion Equations," Nucl. Phys. B528 (1998) 648-682, arXiv:cond-mat/9801158 [cond-mat].

[12] A. Jerez, N. Andrei, and G. Zaránd, "Solution of the Multichannel Coqblin-Schrieffer Impurity Model and Application to Multilevel Systems," Phys. Rev. B58 (1998) 3814-3841, arXiv: cond-mat/9803137 [cond-mat].

[13] P. Coleman, "Mixed valence as an almost broken symmetry," Phys. Rev. B 35 (Apr, 1987) 5072-5116.

[14] Bickers, N., "Review of Techniques in the Large-N Expansion for Dilute Magnetic Alloys," Rev. Mod. Phys. 59 (Oct, 1987) 845-939.

[15] O. Parcollet and A. Georges, "Transition from Overscreening to Underscreening in the Multichannel Kondo Model: Exact Solution at Large N," Phys. Rev. Lett. 79 (1997) 4665-4668, arXiv: cond-mat/9707337 [cond-mat].

[16] O. Parcollet, A. Georges, G. Kotliar, and A. Sengupta, "Overscreened Multi-channel SU(N) Kondo Model: Large-N Solution and Conformal Field Theory," Phys. Rev. B58 (Aug., 1998) 3794-3813, arXiv: cond-mat/9711192.

[17] P. Coleman, "Heavy Fermions: Electrons at the Edge of Magnetism," in Handbook of Magnetism and Advanced Magnetic Materials: Fundamentals and Theory, Kronmuller and Parkin, eds., vol. 1, pp. 95-148. John Wiley and Sons, 2007. [arxiv:cond-mat/0612006].

[18] P. Coleman, "Heavy Fermions and the Kondo Lattice: a 21st Century Perspective," ArXiv e-prints (Sept., 2015), arXiv:1509.05769 [cond-mat.str-el].

[19] I. Affleck, "A Current Algebra Approach To The Kondo Effect," Nucl. Phys. B336 (1990) 517.

[20] I. Affleck and A. Ludwig, "The Kondo Effect, Conformal Field Theory and Fusion Rules," Nucl.Phys. B352 (1991) 849-862.

[21] I. Affleck and A. Ludwig, "Critical Theory of Overscreened Kondo Fixed Points," 
Nucl.Phys. B360 (1991) 641-696.

[22] I. Affleck and A. W. Ludwig, "Universal noninteger 'ground state degeneracy' in critical quantum systems," Phys.Rev.Lett. 67 (1991) 161-164.

[23] I. Affleck and A. Ludwig, "Exact Conformal-field-theory Results on the Multichannel Kondo Effect: Single-fermion Green's function, Self-energy, and Resistivity," Phys.Rev. B48 (1993) 7297-7321.

[24] I. Affleck, "Conformal field theory approach to the Kondo effect," Acta Phys.Polon. B26 (1995) 1869-1932, arXiv: cond-mat/9512099 [cond-mat].

[25] A. Hewson, "The Kondo Model to Heavy Fermions," Cambridge University Press (1993).

[26] D. L. Cox and A. Zawadowski, "Exotic Kondo Effects in Metals: Magnetic Ions in a Crystalline Electric Field and Tunnelling Centres," Advances in Physics $\mathbf{4 7}$ no. 5, (1998) 599-942, arxiv: cond-mat/9704103.

[27] E. S. Sørensen, M.-S. Chang, N. Laflorencie, and I. Affleck, "Impurity entanglement entropy and the Kondo screening cloud," Journal of Statistical Mechanics: Theory and Experiment 2007 no. 01, (2007) L01001. http://stacks.iop.org/1742-5468/2007/i=01/a=L01001.

[28] I. Affleck, N. Laflorencie, and E. S. Sørensen, "Entanglement entropy in quantum impurity systems and systems with boundaries," Journal of Physics A: Mathematical and Theoretical 42 no. 50, (2009) 504009. http://stacks.iop.org/1751-8121/42/i=50/a=504009.

[29] E. S. Sørensen, M.-S. Chang, N. Laflorencie, and I. Affleck, "Quantum impurity entanglement," Journal of Statistical Mechanics: Theory and Experiment 2007 no. 08, (2007) P08003. http://stacks .iop.org/1742-5468/2007/i=08/a=P08003.

[30] E. Eriksson and H. Johannesson, "Impurity entanglement entropy in Kondo systems from conformal field theory," Phys. Rev. B 84 (Jul, 2011) 041107.

[31] C. Latta, F. Haupt, M. Hanl, A. Weichselbaum, M. Claassen, W. Wuester, P. Fallahi, S. Faelt, L. Glazman, J. von Delft, H. E. Türeci, and A. Imamoglu, "Quantum quench of Kondo correlations in optical absorption," Nature 474 (2011) 627-630, arXiv: 1102.3982.

[32] I. Affleck, "The Kondo screening cloud: what it is and how to observe it," ArXiv e-prints (Nov., 2009), arXiv:0911.2209 [cond-mat.mes-hall].

[33] J. M. Maldacena, "The Large N limit of superconformal field theories and supergravity," Int.J.Theor.Phys. 38 (1999) 1113-1133, arXiv:hep-th/9711200 [hep-th].

[34] S. Gubser, I. R. Klebanov, and A. M. Polyakov, "Gauge theory correlators from noncritical string theory," Phys.Lett. B428 (1998) 105-114, arXiv: hep-th/9802109 [hep-th]. 
[35] E. Witten, "Anti-de Sitter space and holography," Adv.Theor.Math.Phys. 2 (1998) 253-291, arXiv:hep-th/9802150 [hep-th].

[36] O. Aharony, S. S. Gubser, J. M. Maldacena, H. Ooguri, and Y. Oz, "Large N field theories, string theory and gravity," Phys. Rept. 323 (2000) 183-386, arXiv:hep-th/9905111 [hep-th].

[37] O. Aharony, O. Bergman, D. L. Jafferis, and J. Maldacena, "N=6 superconformal Chern-Simons-matter theories, M2-branes and their gravity duals," JHEP 10 (2008) 091, arXiv:0806.1218 [hep-th].

[38] S. Kachru, A. Karch, and S. Yaida, "Holographic Lattices, Dimers, and Glasses," Phys. Rev. D81 (2010) 026007, arXiv:0909.2639 [hep-th].

[39] S. Kachru, A. Karch, and S. Yaida, "Adventures in Holographic Dimer Models," New J. Phys. 13 (2011) 035004, arXiv:1009.3268 [hep-th].

[40] A. Faraggi and L. A. Pando Zayas, "The Spectrum of Excitations of Holographic Wilson Loops," JHEP 05 (2011) 018, arXiv:1101.5145 [hep-th].

[41] K. Jensen, S. Kachru, A. Karch, J. Polchinski, and E. Silverstein, "Towards a holographic marginal Fermi liquid," Phys. Rev. D84 (2011) 126002, arXiv:1105.1772 [hep-th].

[42] N. Karaiskos, K. Sfetsos, and E. Tsatis, "Brane embeddings in sphere submanifolds," Class. Quant. Grav. 29 (2012) 025011, arXiv:1106.1200 [hep-th].

[43] S. Harrison, S. Kachru, and G. Torroba, "A maximally supersymmetric Kondo model," Class. Quant. Grav. 29 (2012) 194005, arXiv:1110.5325 [hep-th].

[44] P. Benincasa and A. V. Ramallo, "Fermionic impurities in Chern-Simons-matter theories," JHEP 02 (2012) 076, arXiv:1112.4669 [hep-th].

[45] P. Benincasa and A. V. Ramallo, "Holographic Kondo Model in Various Dimensions," JHEP 06 (2012) 133, arXiv:1204.6290 [hep-th].

[46] A. Faraggi, W. Mueck, and L. A. Pando Zayas, "One-loop Effective Action of the Holographic Antisymmetric Wilson Loop," Phys. Rev. D85 (2012) 106015, arXiv:1112.5028 [hep-th].

[47] G. Itsios, K. Sfetsos, and D. Zoakos, "Fermionic impurities in the unquenched ABJM," JHEP 01 (2013) 038, arXiv:1209.6617 [hep-th].

[48] J. Erdmenger, C. Hoyos, A. O'Bannon, and J. Wu, "A Holographic Model of the Kondo Effect," JHEP 1312 (2013) 086, arXiv:1310.3271 [hep-th].

[49] J. M. Maldacena, "Wilson Loops in Large N Field Theories," Phys.Rev.Lett. 80 (1998) 4859-4862, arXiv:hep-th/9803002 [hep-th].

[50] S.-J. Rey and J.-T. Yee, "Macroscopic Strings as Heavy Quarks in Large N Gauge Theory and Anti-de Sitter Supergravity," Eur.Phys.J. C22 (2001) 379-394, arXiv:hep-th/9803001 [hep-th]. 
[51] J. M. Camino, A. Paredes, and A. V. Ramallo, "Stable wrapped branes," JHEP 05 (2001) 011, arXiv: hep-th/0104082 [hep-th].

[52] S. Yamaguchi, "Wilson Loops of Anti-symmetric Representation and D5-branes," JHEP 0605 (2006) 037, arXiv:hep-th/0603208 [hep-th].

[53] J. Gomis and F. Passerini, "Holographic Wilson Loops," JHEP 08 (2006) 074. arXiv:hep-th/0604007 [hep-th].

[54] J. Gomis and F. Passerini, "Wilson Loops as D3-Branes," JHEP 0701 (2007) 097, arXiv:hep-th/0612022 [hep-th].

[55] O. Parcollet, A. Georges, G. Kotliar, and A. Sengupta, "Overscreened multichannel $\mathrm{SU}(N)$ Kondo model: Large- $N$ solution and conformal field theory," Phys. Rev. B $\mathbf{5 8}$ (Aug, 1998) 3794-3813, arXiv: cond-mat/9711192 [cond-mat].

[56] T. Senthil, S. Sachdev, and M. Vojta, "Fractionalized Fermi Liquids," Phys. Rev. Lett. 90 (May, 2003) 216403.

[57] E. Witten, "Multitrace operators, boundary conditions, and AdS / CFT correspondence," arXiv:hep-th/0112258 [hep-th].

[58] S. Ryu and T. Takayanagi, "Holographic derivation of entanglement entropy from AdS/CFT," Phys.Rev.Lett. 96 (2006) 181602, arXiv:hep-th/0603001 [hep-th].

[59] S. Ryu and T. Takayanagi, "Aspects of Holographic Entanglement Entropy," JHEP 0608 (2006) 045, arXiv:hep-th/0605073 [hep-th].

[60] T. Takayanagi, "Holographic Dual of BCFT," Phys.Rev.Lett. 107 (2011) 101602, arXiv: 1105.5165 [hep-th].

[61] M. Fujita, T. Takayanagi, and E. Tonni, "Aspects of AdS/BCFT," JHEP 1111 (2011) 043, arXiv:1108.5152 [hep-th].

[62] M. Nozaki, T. Takayanagi, and T. Ugajin, "Central Charges for BCFTs and Holography," JHEP 1206 (2012) 066, arXiv:1205.1573 [hep-th].

[63] J. Erdmenger, M. Flory, and M.-N. Newrzella, "Bending branes for DCFT in two dimensions," JHEP 1501 (2015) 058, arXiv:1410.7811 [hep-th].

[64] D. Friedan and A. Konechny, "On the boundary entropy of one-dimensional quantum systems at low temperature," Phys.Rev.Lett. 93 (2004) 030402, arXiv:hep-th/0312197 [hep-th].

[65] H.-Q. Zhou, T. Barthel, J. O. Fjærestad, and U. Schollwöck, "Entanglement and boundary critical phenomena," Phys. Rev. A 74 (Nov, 2006) 050305.

[66] R. A. Janik, J. Jankowski, and P. Witkowski, "Conformal defects in supergravity backreacted Dirac delta sources," JHEP 07 (2015) 050, arXiv: 1503.08459 [hep-th].

[67] L. Susskind, "Computational Complexity and Black Hole Horizons," arXiv:1402.5674 [hep-th]. 
[68] L. Susskind, "Addendum to Computational Complexity and Black Hole Horizons," arXiv:1403.5695 [hep-th].

[69] M. Alishahiha, "Holographic Complexity," arXiv:1509.06614 [hep-th].

[70] A. R. Brown, D. A. Roberts, L. Susskind, B. Swingle, and Y. Zhao, "Complexity Equals Action," arXiv:1509.07876 [hep-th].

[71] M. Miyaji, T. Numasawa, N. Shiba, T. Takayanagi, and K. Watanabe, "Gravity Dual of Quantum Information Metric," arXiv:1507.07555 [hep-th].

[72] K. Skenderis and M. Taylor, "Branes in AdS and p p wave space-times," JHEP 06 (2002) 025, arXiv:hep-th/0204054 [hep-th].

[73] J. A. Harvey and A. B. Royston, "Localized modes at a D-brane-O-plane intersection and heterotic Alice atrings," JHEP 04 (2008) 018, arXiv:0709.1482 [hep-th].

[74] J. A. Harvey and A. B. Royston, "Gauge/Gravity duality with a chiral $N=(0,8)$ string defect," JHEP 08 (2008) 006, arXiv:0804.2854 [hep-th].

[75] E. I. Buchbinder, J. Gomis, and F. Passerini, "Holographic gauge theories in background fields and surface operators," JHEP 12 (2007) 101, arXiv:0710.5170 [hep-th].

[76] M. Banados, C. Teitelboim, and J. Zanelli, "The Black hole in three-dimensional space-time," Phys.Rev.Lett. 69 (1992) 1849-1851, arXiv:hep-th/9204099 [hep-th].

[77] M. Banados, M. Henneaux, C. Teitelboim, and J. Zanelli, "Geometry of the $(2+1)$ black hole," Phys.Rev. D48 (1993) 1506-1525, arXiv:gr-qc/9302012 [gr-qc].

[78] A. O'Bannon, I. Papadimitriou, and J. Probst, "A Holographic Two-Impurity Kondo Model," arXiv:1510.08123 [hep-th].

[79] W. Israel, "Singular hypersurfaces and thin shells in general relativity," Nuovo Cim. B44S10 (1966) 1.

[80] M. Alishahiha and R. Fareghbal, "Boundary CFT from Holography," Phys.Rev. D84 (2011) 106002, arXiv:1108.5607 [hep-th].

[81] M. Setare and V. Kamali, "Correlation functions of BCFT," arXiv:1109.3849 [hep-th].

[82] Y. Kwon, S. Nam, J.-D. Park, and S.-H. Yi, "AdS/BCFT Correspondence for Higher Curvature Gravity: An Example," JHEP 1206 (2012) 119, arXiv:1201.1988 [hep-th].

[83] M. Fujita, M. Kaminski, and A. Karch, "SL(2,Z) Action on AdS/BCFT and Hall Conductivities," JHEP 1207 (2012) 150, arXiv:1204.0012 [hep-th].

[84] Y. Nakayama, "Is boundary conformal in CFT?," Phys.Rev. D87 no. 4, (2013) 046005, arXiv:1210.6439 [hep-th]

[85] D. Melnikov, E. Orazi, and P. Sodano, "On the AdS/BCFT Approach to Quantum Hall Systems," JHEP 1305 (2013) 116, arXiv:1211.1416 [hep-th]. 
[86] A. F. Astaneh and A. E. Mosaffa, "Quantum Local Quench, AdS/BCFT and Yo-Yo String," arXiv:1405.5469 [hep-th].

[87] J. M. Magán, D. Melnikov, and M. R. O. Silva, "Black Holes in AdS/BCFT and Fluid/Gravity Correspondence," arXiv:1408.2580 [hep-th].

[88] L. Randall and R. Sundrum, "An Alternative to compactification," Phys.Rev.Lett. 83 (1999) 4690-4693, arXiv:hep-th/9906064 [hep-th].

[89] A. Karch and L. Randall, "Locally localized gravity," JHEP 0105 (2001) 008, arXiv:hep-th/0011156 [hep-th].

[90] A. Karch and L. Randall, "Open and closed string interpretation of SUSY CFT's on branes with boundaries," JHEP 0106 (2001) 063, arXiv: hep-th/0105132 [hep-th]

[91] R. A. Battye and B. Carter, "Generic junction conditions in brane world scenarios," Phys.Lett. B509 (2001) 331-336, arXiv: hep-th/0101061 [hep-th].

[92] P. Kraus, "Dynamics of anti-de Sitter domain walls," JHEP 12 (1999) 011, arXiv:hep-th/9910149 [hep-th].

[93] P. Bowcock, C. Charmousis, and R. Gregory, "General brane cosmologies and their global space-time structure," Class. Quant. Grav. 17 (2000) 4745-4764, arXiv:hep-th/0007177 [hep-th].

[94] P. Breitenlohner and D. Z. Freedman, "Stability in Gauged Extended Supergravity," Annals Phys. 144 (1982) 249

[95] T. Azeyanagi, A. Karch, T. Takayanagi, and E. G. Thompson, "Holographic calculation of boundary entropy," JHEP 0803 (2008) 054-054, arXiv:0712.1850 [hep-th].

[96] S. Yamaguchi, "Holographic RG flow on the defect and g theorem," JHEP 10 (2002) 002, arXiv:hep-th/0207171 [hep-th].

[97] G. T. Horowitz and M. M. Roberts, "Zero Temperature Limit of Holographic Superconductors," JHEP 11 (2009) 015, arXiv:0908.3677 [hep-th].

[98] O. J. Dias, G. T. Horowitz, N. Iqbal, and J. E. Santos, "Vortices in holographic superfluids and superconductors as conformal defects," JHEP 1404 (2014) 096, arXiv:1311.3673 [hep-th]

[99] G. T. Horowitz, N. Iqbal, J. E. Santos, and B. Way, "Hovering Black Holes from Charged Defects," Class. Quant. Grav. 32 (2015) 105001, arXiv:1412.1830 [hep-th].

[100] A. Bayat, P. Sodano, and S. Bose, "Negativity as the entanglement measure to probe the Kondo regime in the spin-chain Kondo model," Phys. Rev. B $\mathbf{8 1}$ (Feb, 2010) 064429.

[101] A. Bayat, H. Johanesson, S. Bose, and P. Sodano, "Order Parameter for Impurity Systems at Quantum Criticality," Nature Communications 5 (2014) 3784 , arXiv: 1404.0828 .

[102] S.-S. B. Lee, J. Park, and H.-S. Sim, "Macroscopic Quantum Entanglement of a Kondo 
Cloud at Finite Temperature," Phys. Rev. Lett. 114 (Feb, 2015) 057203.

[103] K. T. Law, C. Y. Seng, P. A. Lee, and T. K. Ng, "Quantum dot in a two-dimensional topological insulator: The two-channel Kondo fixed point," Phys. Rev. B 81 (Jan, 2010) 041305 .

[104] K. Hattori, K. Itakura, S. Ozaki, and S. Yasui, "QCD Kondo effect: quark matter with heavy-flavor impurities," arXiv:1504.07619 [hep-ph].

[105] H. Matsueda, "Multiscale Entanglement Renormalization Ansatz for Kondo Problem," ArXiv e-prints (Aug., 2012), arXiv:1208.2872 [cond-mat.stat-mech].

[106] A. Antillon, J. Escalona, and M. Torres, "Vortices and domain walls in a Chern-Simons theory with magnetic moment interaction," Phys. Rev. D55 (1997) 6327-6338, arXiv:hep-th/9702118 [hep-th].

[107] M. Torres, "Domain walls in a Chern-Simons theory," in Solitons: Properties, dynamics, interactions, applications. Proceedings, Workshop, Kingston, Canada, July 20-26, 1997. 1997. arXiv:hep-th/9710113 [hep-th]. 